\title{
Flow Distortion Measurements in Convoluted Aero Engine Intakes
}

\author{
Pavlos K. Zachos ${ }^{1}$, David G. MacManus ${ }^{2}$ and Nicola Chiereghin ${ }^{3}$ \\ Propulsion Engineering Centre, School of Aerospace, Transport and Manufacturing, Cranfield University, \\ Cranfield, MK43 0AL, UK
}

The unsteady flowfields generated by convoluted aero engine intakes are major sources of instabilities that can compromise the performance of the downstream turbomachinery components. Hence, there exists a need for high spatial and temporal resolution measurements that will allow a greater understanding of the aerodynamics. Stereoscopic Particle Image Velocimetry is capable of providing such fidelity but its application has been limited previously as the optical access through cylindrical ducts for air flow measurements constitutes a notable pitfall for this type of measurements. This paper presents a suite of S-PIV measurements and flow field analysis in terms of snapshot, statistical and time-averaged measurements for two S-duct configurations across a range of inlet Mach numbers. The flow assessments comprise effects of inlet Mach number and S-duct centerline offset distance. Overall, the work demonstrates the feasibility of using S-PIV techniques for determining the complex flow field at the exit of convoluted intakes with at least two orders of magnitude higher spatial resolution than the traditional pressure rake measurements allow. Analysis of the conventional distortion descriptors quantifies the dependency upon the S-duct configuration and highlights that the more aggressive duct generates twice the levels of swirl distortion than the low offset one. The analysis also shows a weak dependency of the distortion descriptor magnitude upon the inlet Mach number across the entire range of Mach numbers tested. A statistical assessment of the unsteady distortion history over the data acquisition time highlights the dominant swirl patterns of the two configurations. Such an advancement in measurement capability enables a significantly more substantial steady and unsteady flow analyses and highlights the benefits of synchronous high resolution three component velocity measurements to unlock the aerodynamics of complex engine-intake systems.

\section{Nomenclature}

$\begin{array}{lll}\text { List of symbols } & & \\ A & = & \text { Area } \\ D & = & \text { Diameter } \\ H & = & \text { Offset } \\ k & = & \text { Swirl pairs index } \\ L & = & \text { Tength } \\ p_{0} & = & \text { Dotal pressure } \\ q & = & \text { Cynamic head } \\ r, \theta, z & & \end{array}$

\footnotetext{
${ }^{1}$ Lecturer in Aerodynamics, Propulsion Engineering Centre, Cranfield University, M43 0AL, UK, AIAA Member.

${ }^{2}$ Senior Lecturer in Aerodynamics, Propulsion Engineering Centre, Cranfield University, M43 0AL, UK, AIAA Member.

${ }^{3}$ Doctoral Researcher, Propulsion Engineering Centre, Cranfield University, M43 0AL, UK.
} 


$\begin{array}{lll}R e_{D} & = & \text { Diameter based Reynolds number } \\ s_{s} & = & \text { Statistical skewness } \\ s_{k} & = & \text { Statistical kurtosis } \\ u_{r}, u_{\theta}, w & = & \text { Velocity vector components } \\ u_{r}, u_{\theta}, w & = & \text { Velocity fluctuations } \\ U_{r}, U_{\theta}, W & = & \text { Area average of the time average velocity components } \\ x, y, z & = & \text { Cartesian system of reference coordinates }\end{array}$

\section{Abbreviations}

AIP
CDI
CFD
DC $(\theta)$
DEHS
RANS
RDI
SAE
SC $(\theta)$
SD
SI
SP
S-PIV
SS
TKE
TI

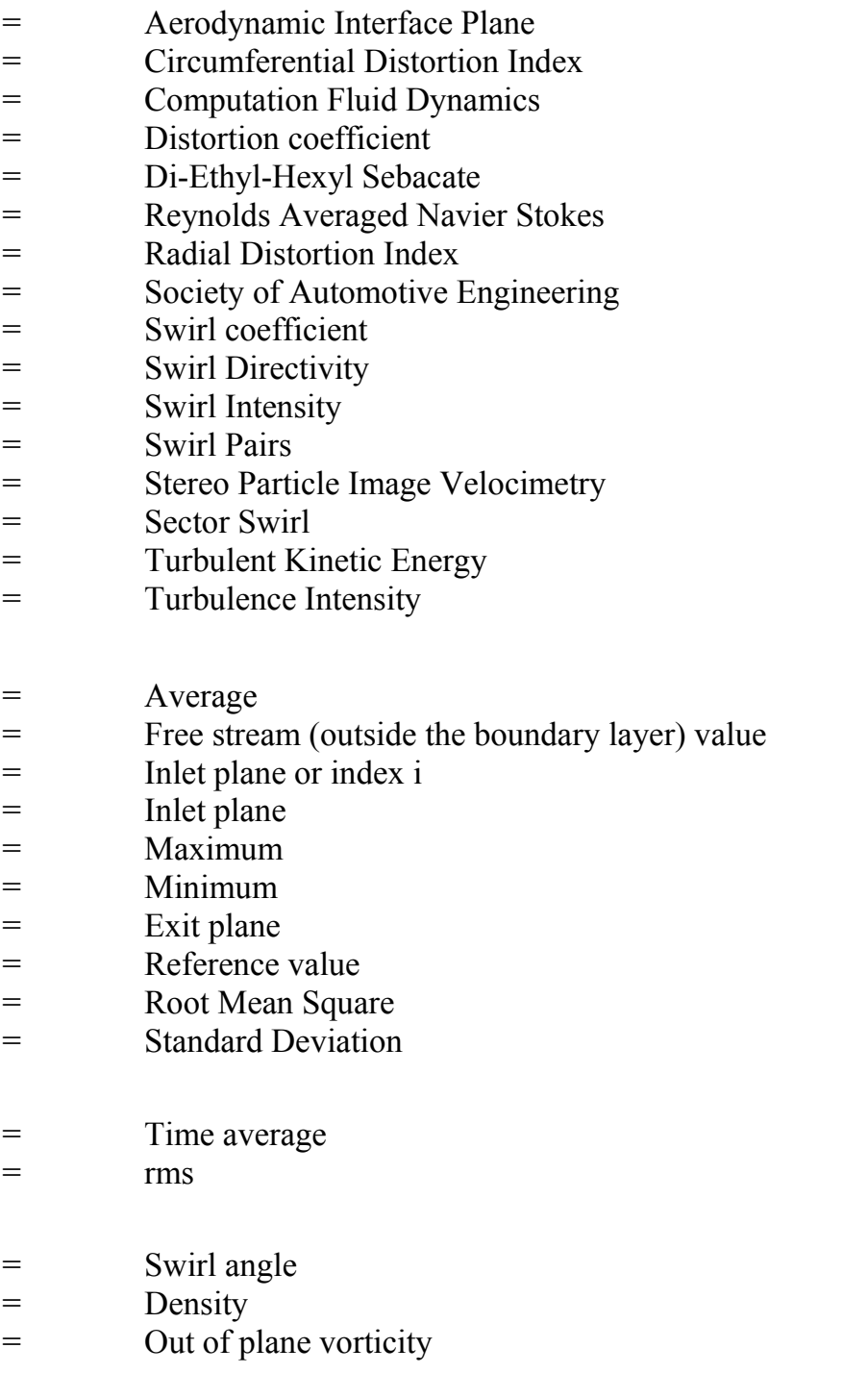

\section{Subscripts}

avg, mean
$e$
$i$
in
$\max$
min
out
ref
rms
std

\section{Operators}

$<>$

$\sigma$

\section{Introduction}

$\mathrm{W}_{\mathrm{c}}^{\mathrm{i}}$

ith the drive towards shorter and more complex intakes for advanced propulsion system installations, the coupling between the intake and fan becomes more critical. Intake flow distortion can be especially severe for convoluted ducts relevant to close-coupled engine installation arrangements. This is of interest to a range of applications including future civil aircraft configurations which may feature novel propulsion integration configurations ${ }^{1,2}$. These designs with unconventional intakes are susceptible to flow separations, which lead to complex total pressure and swirl flow fields, significant vortical regions and large unsteady flow perturbations at the aerodynamic interface plane (AIP $)^{3-5}$. Total pressure and swirl distortions can be generated by flow separations within the intake which may result in low momentum flow regions or swirling flow which is either co- or contra rotating with respect to the compression system and affects flow incidence onto the blade incidence. As a result, complex total pressure and swirl flow fields are generated at the AIP, which, in turn, affects the flow capacity as 
well as the pressure rise and the surge margin of the compression system. An illustration of this situation is shown in Fig. 1 where the introduction of co-rotating swirl, along with total-pressure distortion, slightly decreased the stability margin compared to total-pressure distortion alone ${ }^{6}$. However, counter-rotating swirl with total-pressure distortion greatly reduced the stability margin. It has been observed that counter-rotating swirl in conjunction with total pressure distortion can half the surge margin of the compression system.

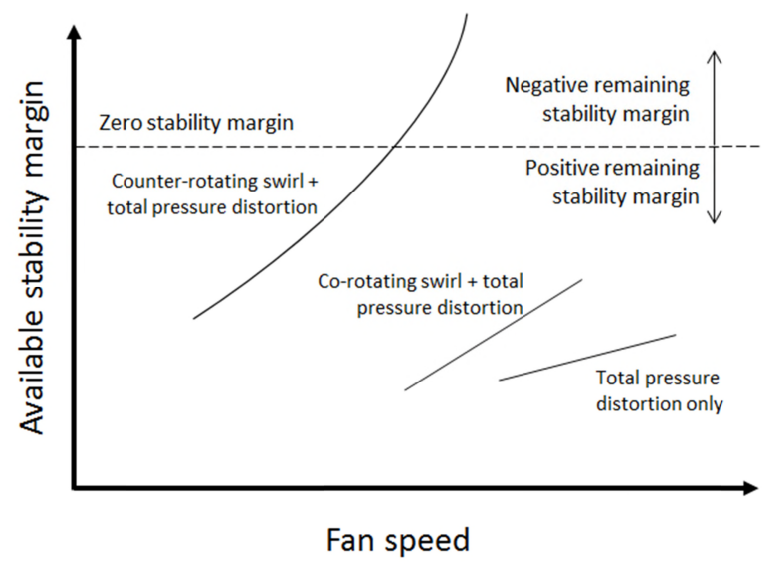

Figure 1. Effects of swirl and total pressure distortion on engine stability ${ }^{6}$.

For the design of convoluted engine intakes, one of the design drivers is the minimization of the distortion that can result in a non-uniform distribution of swirl angle and total pressure across the AIP and which may adversely affect the engine stability or operation. The time averaged total pressure and swirl angle distributions across the exit plane of a typical diffusing S-duct ${ }^{7}$ is shown in Fig. 2 as an example of the flow non-uniformities that arise in such configurations and are presented at the AIP. This shows a result of a computational fluid dynamics (CFD) study using a steady Reynolds Averaged Navier Stokes method ${ }^{7}$. The area ratio of the S-duct was $\mathrm{A}_{\text {out }} / \mathrm{A}_{\text {in }}=1.52$, the offset to inlet diameter ratio $\mathrm{H} / \mathrm{D}_{\mathrm{i}}$ was 1.34 and the length to inlet diameter ratio $\mathrm{L} / \mathrm{D}_{\mathrm{i}}$ was 5.0. Visualization of the computed streaklines on the duct surface showed a separation bubble on the lower part of the AIP containing two vortical structures which arise from the development of the classical secondary flows around the first part of the duct. Crossflow plane measurements reported by Wellborn et al ${ }^{3}$, using a five-hole probe, showed that the timeaveraged flow field comprised a pair of streamwise vortices at the outlet of the S-duct associated with a low pressure zone on the lower part of the plane. Such a simultaneous presence of total pressure and swirl at the inlet of the compressor has previously been demonstrated to be a source of loss in surge margin, while it increases the possibility of stall inception ${ }^{6,8}$. High bandwidth pressure measurements in two similar geometries ${ }^{4,5}$ highlighted a

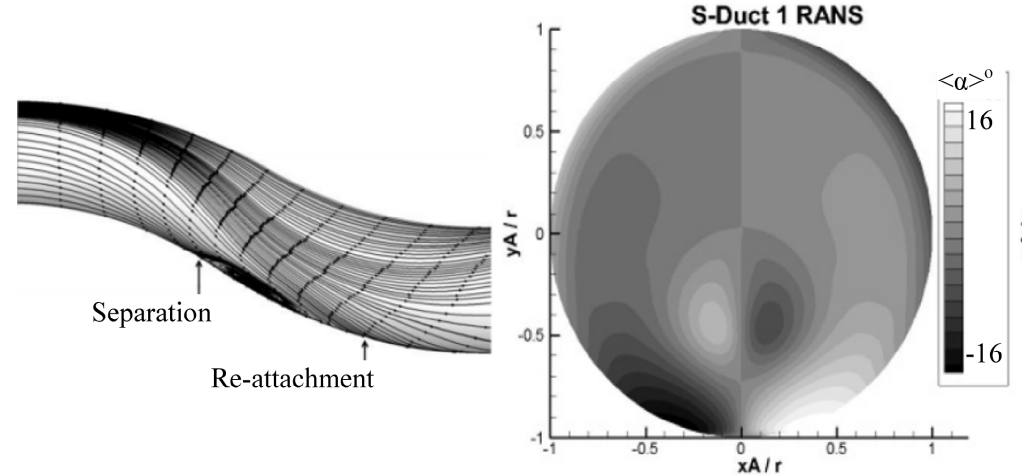

a) b)

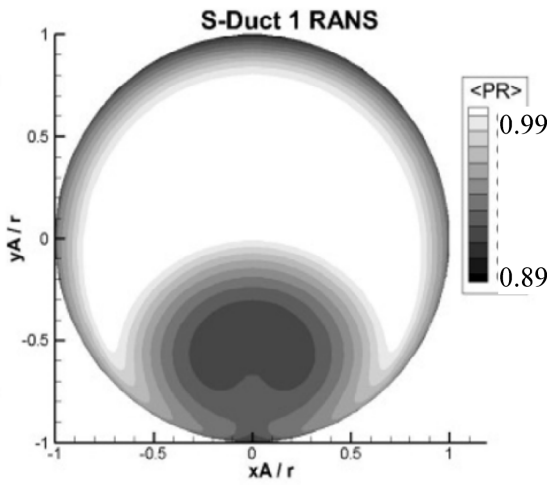

c)

Figure 2. Example of the flow field for a convoluted S-duct calculated using a steady RANS simulation. (a) Surface streaklines (b) swirl angle distribution and (c) total pressure distribution at the AIP ${ }^{7}$.

notable instability in the separation zone. This instability was found to produce oscillations of the flow distortions at 
the exit plane, which is an additional source of loss in performance and operability for the compression system. For swirl distributions almost no publicly available data exist to support the understanding of the underlying unsteady swirl distortion characteristics. However, from industrial experience, the need to consider swirl distributions during operability assessments is evident across a wide range of aircraft turbine applications ${ }^{6}$.

From an industrial perspective, to systematically quantify the different kinds of distortion, various descriptors have been introduced by engine manufacturers and engineering bodies ${ }^{6,8,10,15}$. Current industry practice for the quantification of distorted flow fields for compressor or fan systems typically relies on steady state flow experimental tests which subsequently allow characterization of distortion via the appropriate descriptors. For example, a standard measurement arrangement for advanced engine intakes uses a total pressure rake at the Aerodynamic Interface Plane (AIP) comprising an array of 8 spokes with 5 probes each ${ }^{6}$. Flow data from these positions is then used to describe distortion of total pressure with a similar approach, with typically less spatial resolution for total temperature and swirl non-uniformities. Although these low frequency, time-averaged measurements may be supplemented with a set of unsteady pressure or swirl measurements it is considered as being unable to capture the detailed unsteady behavior of the flow ${ }^{6,9}$. This is mainly due to its low spatial and temporal resolution which is insufficient to capture the complex nature of the generated flow fields.

Nevertheless, a range of total pressure based distortion descriptors such as CDI, RDI and DC(60) are typically considered. DC(60) is given by the difference between the average total pressure and the lowest average total pressure in a sector of $60^{\circ}$ angle and non-dimensionalized by the mean dynamic head q of the $\mathrm{AIP}^{10}$.

$$
\operatorname{DC}(60)=\frac{\mathrm{p}_{0, \mathrm{avg}}-\mathrm{p}_{0,60^{\circ} \mathrm{avg}}}{\mathrm{q}}
$$

where $\mathrm{p}_{\mathrm{o} \text { avg }}$ is the mean total pressure and $\mathrm{p}_{60^{\circ}}$ avg is the mean total pressure measure in a sector of 60 degrees. The circumferential distortion index (CDI) assesses the uniformity of the circumferential total pressure distribution and is defined as:

$$
\mathrm{CDI}=\operatorname{Max}_{\mathrm{i}=1}^{\mathrm{n}_{\text {radius }}-1}\left(0.5\left[\frac{\mathrm{p}_{0 \mathrm{i}}-\mathrm{p}_{0 \mathrm{~min}_{\mathrm{i}}}}{\mathrm{p}_{0, \text { avg }}}+\frac{\mathrm{p}_{0 \mathrm{i}+1}-\mathrm{p}_{0 \mathrm{~min}_{\mathrm{i}+1}}}{\mathrm{p}_{0, \mathrm{avg}}}\right]\right)
$$

where $p_{0 a v g}$ is the average total pressure, $p_{0 \mathrm{i}}$ the average total pressure of the pressure distribution of the $\mathrm{i}$-th ring and $\mathrm{p}_{\text {min }_{\mathrm{i}}}$ the minimal pressure of the $i$-th ring ${ }^{10}$. Finally the radial distortion can be assessed by the radial distortion index (RDI). The formula follows the same logic as CDI and is defined as ${ }^{10}$ :

$$
\mathrm{RDI}=\operatorname{Max}\left(\frac{\mathrm{p}_{0, \text { avg }}-\mathrm{p}_{0, \text { inner ring }}}{\mathrm{p}_{0, \mathrm{avg}}}, \frac{\mathrm{p}_{0, \mathrm{avg}}-\mathrm{p}_{0, \text { outer ring }}}{\mathrm{p}_{0, \mathrm{avg}}}\right)
$$

with $\mathrm{p}_{\text {inner ring }}$ the average total pressure of the pressure distribution of the inner ring and $\mathrm{p}_{0, \text { outer ring }}$ the average total pressure at the outer ring.

A key aspect to achieving substantially higher spatial resolution is a measurement system that can provide sufficient spatially and temporally rich measurements of the detailed flow distortion characteristics. The low spatial resolution that conventional distortion measurements provide can be addressed by Particle Image Velocimetry methods (S-PIV), which, relative to an $8 \times 5$ pressure rake, has the potential to provide 200-300 times higher spatial resolution across a measurement plane. Although S-PIV is a relatively mature measurement method, there has been very limited previous work which measures the full cross plane of an intake. This is a key requirement for inlet flow distortion quantification. Previous experiments for cylindrical ducts with water reported by Doorne et al ${ }^{11}$ proved the feasibility of S-PIV techniques to measure flows with large out-of-plane velocity components Doorne et $\mathrm{al}^{11}$ and Nelson et $\mathrm{al}^{12}$ highlighted the complexities of S-PIV through cylindrical ducts and quantified some of the underlying challenges associated with it. These tests showed that the curved cylindrical walls of the test section cause additional optical distortions which impose uncertainties onto the mapping functions especially close to the boundaries. These errors can be accounted for through a mis-registration correction algorithm. The importance of laser light management was also highlighted to avoid excessive secondary light scattering and reflections by the cylindrical walls which increase up to $60 \%$ the measurement noise ${ }^{12}$.

The aim of this paper is to use Stereoscopic Particle Image Velocimetry (S-PIV) to provide a step change in the measurement and analysis of the distorted velocity flow field generated by a complex aero-engine intake. For this 
reason a bespoke wind tunnel was designed and built to facilitate the installation of various convoluted engine intakes (S-ducts). The practical challenges associated with the application of this technique within confined, highly distorted internal flows are assessed along with aspects on the measurement uncertainties. Measurement results are presented, while the steady and unsteady characteristics of the flow are studied through a statistical analysis of the key velocity parameters and swirl descriptors.

\section{Experimental setup and methods}

\section{A. Experimental facility}

The experiments were conducted at a bespoke facility at Cranfield University which was designed to accommodate a range of convoluted ducts and to allow good optical access to enable S-PIV measurements at the crossflow exit plane of the intake ducts. The general arrangement of the test facility is shown in Fig. 3. The facility comprises a bell-mouth intake followed by a flow conditioning section of $200 \mathrm{~mm}$ diameter which contains a honeycomb mesh which is $100 \mathrm{~mm}$ long and with hexagonal cell sizes of $6 \mathrm{~mm}$ diagonal length. Aft of the intake throat and ahead of the honeycomb section, twelve static pressure tappings are equispaced circumferentially and, in conjunction with the intake reference total pressure, are used to set the rig operating point. Downstream of the flow conditioner is a constant diameter section of $200 \mathrm{~mm}$ diameter which can accommodate a swirl distortion generator and then a conical nozzle section where the diameter reduces from $200 \mathrm{~mm}$ to $120 \mathrm{~mm}$ over a distance of $200 \mathrm{~mm}$. This is followed by a straight cylindrical section with an inlet diameter of $120 \mathrm{~mm}$ and a length of $300 \mathrm{~mm}$. This leads to the inlet plane of the convoluted ducts with the same inlet diameter of $\mathrm{D}_{\mathrm{i}}=120 \mathrm{~mm}$.

The test rig was designed in a modular way so that the installation of various S-duct geometries can be facilitated. Two convoluted ducts have been design, manufactured and tested. The baseline configuration has an offset-to-length ratio of $\mathrm{H} / \mathrm{D}_{\mathrm{i}}=1.34, \mathrm{H} / \mathrm{L}=0.268, \mathrm{~L} / \mathrm{D}_{\mathrm{i}}=5$ and a diffusing area ratio $\mathrm{A}_{\text {out }} / \mathrm{A}_{\text {in }}=1.52$ (Fig. 4). An $\mathrm{S}$ duct with a relatively higher offset has been also tested based on the work of Garnier et al ${ }^{5}$ with a $H / D_{i}=2.44, H / L=$ 0.493 , an $\mathrm{L} / \mathrm{D}_{\text {in }}=5.0$ and the same $\mathrm{A}_{\text {out }} / \mathrm{A}_{\text {in }}=1.52$. For this work the diameter at the aerodynamic interface plane (AIP) is $150 \mathrm{~mm}$ for both configurations. This is similar to previous work that mostly focused on conventional steady and unsteady pressure measurements ${ }^{4,5}$. At the exit of the S-duct a borosilicate glass working section with an inner diameter of $150 \mathrm{~mm}$ and a length of $300 \mathrm{~mm}$ was used. The working section was then linked to a retractable diffusor duct and ultimately to a single stage centrifugal fan. The retractable diffuser provided relatively easy access to the working section for cleaning purposes.

At a location of $0.9 \mathrm{D}_{\mathrm{i}}$ upstream of the duct inlet plane, the circumferential distribution of static pressure is measured using 6 equispaced tappings. The total pressure profile was measured $0.9 \mathrm{D}_{\mathrm{i}}$ upstream the S-duct inlet using a flattened boundary layer Pitot probe which has a dimension of $0.4 \mathrm{~mm}$ by $1.05 \mathrm{~mm}$. The traverse resolution was $0.5 \mathrm{~mm}$. The total pressure profile, in combination with the circumferentially averaged static pressures, were used for the derivation of the inlet Mach number profile across the range of tunnel operating conditions. The inlet Mach number profile was then reduced to a single inlet Mach value using an area weighted averaging process. The uncertainty on the inlet Mach number was assessed by considering the uncertainty elements of the total pressure measurement transducer as well as the errors attributed to the measurement of ambient pressure and total temperature. The deterministic total pressure measurement uncertainties comprise the transducer accuracy as given by the manufacturer, the resolution uncertainty and the calibration uncertainty. The stochastic element was taken into account as the standard deviation of the mean value of the sample which was acquired at $1 \mathrm{kHz}$ for 10 seconds. The test cell ambient pressure was measured by means of a digital manometer. The ambient temperature was measured via a thermocouple. The uncertainties of both instruments were provided by the manufacturers. Using uncertainty propagation analysis, the uncertainty at the inlet Mach number was calculated as $0.27 \pm 0.004$ and $0.6 \pm 0.002$. 


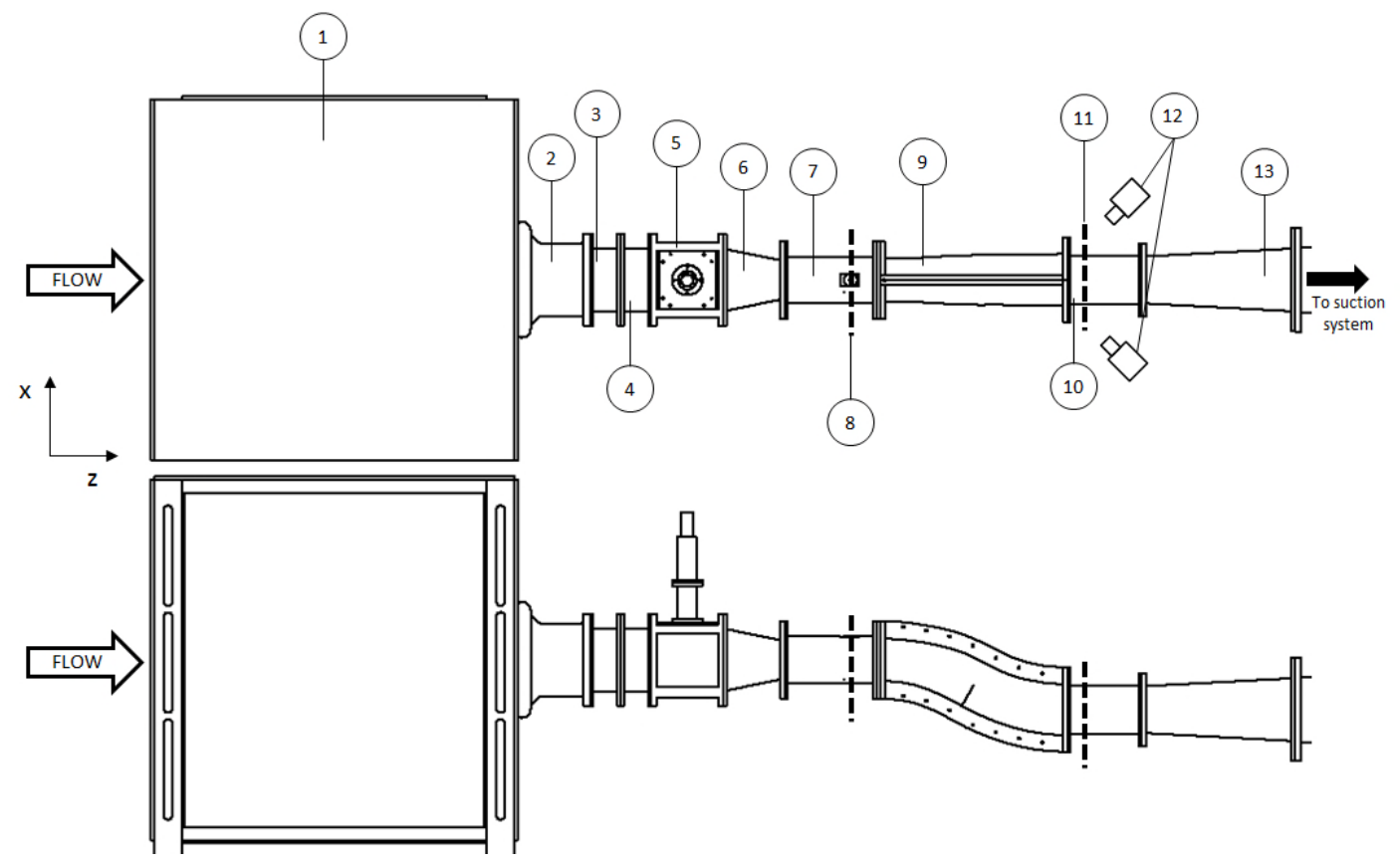

Figure 3. General arrangement of the test rig 1: Seeding chamber, 2: Flare inlet, 3: Flow measurement section, 4: Flow treatment section, 5: Vortex generator 6: Conical nozzle, 7: Straight cylindrical section, 8: Inlet traverse plane at 0.9Di upstream S-duct inlet, 9: S-duct, 10: Glass working section, 11: S-PIV measurement plane at 0.25Dout, 12: PIV cameras, 13: Exhaust diffuser.

\begin{tabular}{ccc}
\hline \hline Parameter & Low offset & High offset \\
\hline $\mathrm{D}_{\mathrm{i}}$ & $121.6 \mathrm{~mm}$ & $121.6 \mathrm{~mm}$ \\
$\mathrm{~A}_{\text {out }} / \mathrm{A}_{\text {in }}$ & 1.52 & 1.52 \\
$\mathrm{H} / \mathrm{D}_{\mathrm{i}}$ & 1.34 & 2.44 \\
$\mathrm{~L}_{\mathrm{i}}$ & 5.0 & 5.0 \\
\hline \hline
\end{tabular}

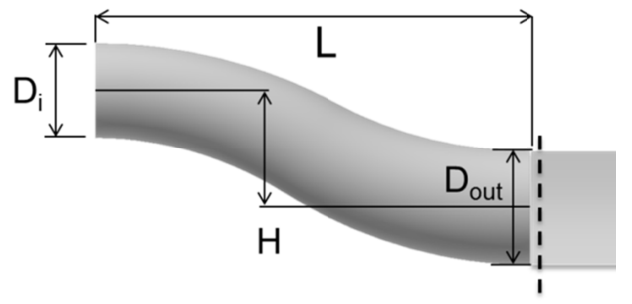

a)

b)

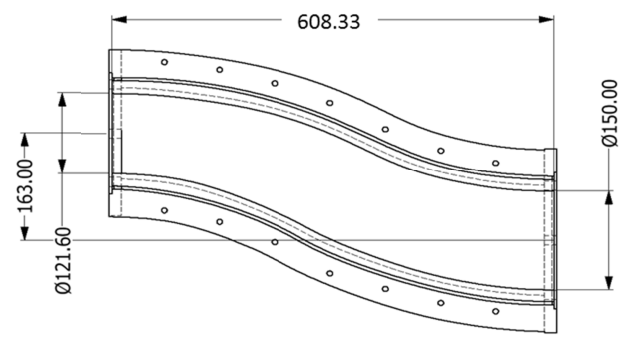

c)

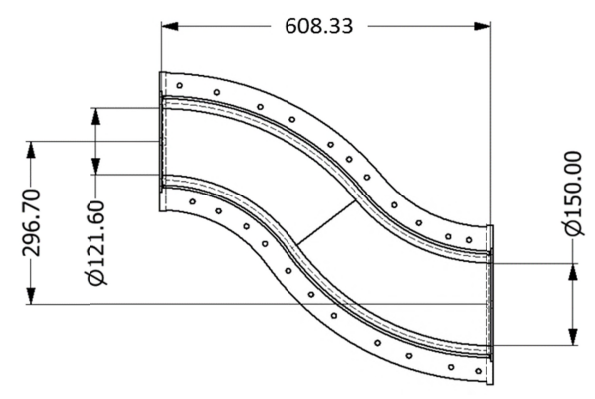

d)

Figure 4. Parametric definition (a) and design parameters for the two tested S-ducts (b). Low offset (c) and high offset configuration (d). 


\section{B. S-PIV methods}

The flow at the AIP was illuminated by a dual cavity frequency-doubled pulsed Nd:YAG laser with a maximum power of $200 \mathrm{~mJ} /$ pulse and an acquisition rate of $7.5 \mathrm{~Hz}$. The amount of light used for the data acquisition was adjusted as required using a motorised attenuator. The laser light sheet was delivered by an articulated light arm, which combines a series of optics to convert the incoming laser beam into a thin light sheet which was adjusted to cover the full AIP area. The delivered laser light sheet had an estimated thickness of $1.5 \mathrm{~mm}$. The laser arm was mounted onto a positioning system that allowed translation of the light sheet parallel to the rig axis, lateral translation as well as 2-axes rotation. This arrangement allows for exact positioning of the light sheet within the region of interest.

Two TSI PowerView Plus cameras were used in a stereoscopic partial side scatter configuration with an approximately $45^{\circ}$ off-axis arrangement (see Fig. 3). The cameras had a 4MP square sensor with an image resolution of 2048 x 2048px. AF 1.8/D Nikkor lenses were employed with a focal length of $50 \mathrm{~mm}$ and the cameras were positioned with a stand-off distance of approximately $600 \mathrm{~mm}$. The camera mounts feature a coarse and a fine 2-axis positioning system that allows the positioning of the cameras along the $\mathrm{x}-\mathrm{z}$ plane. The cameras were positioned along the AIP centreline and therefore the camera alignment and imaging was facilitated by a planar Scheimpflung mechanism. The camera aperture, focus and Scheimpflung setting were all remotely controlled.

Di-Ethyl-Hexyl Sebacate (DEHS) was used for the seeding of the flow. The seeding particles were generated by a Laskin nozzle particle generator. A 10 bar 860 liter pressure vessel was used to feed the seeder with the required airflow for the duration of the tests. The air-seeding mixture is pre-conditioned in a bespoke seeding chamber. The chamber features four solid walls and two open surfaces that each feature a double layer of turbulence mesh. The meshes are designed so that the wires of the external layer generate turbulent flow that is expected to enhance the mixing process while the inner layer damps the flow turbulence levels to provide a homogenous air-seeding cloud which is subsequently ingested by the rig. The turbulence grids are designed based on the guidelines provided by Roach $^{13}$.

The camera spatial calibration at the region of interest was performed using a calibration target featuring a rectangular grid of $10 \mathrm{~mm}$ spaced dots with a $2 \mathrm{~mm}$ diameter. The target plate was placed in the glass working section and the multi plane calibration was performed using a translation along the z-axis of the working section. A five plane calibration was used with an interplane spacing along the $\mathrm{z}$ axis of $0.375 \mathrm{~mm}$.

\section{Image processing and vector evaluation}

The evaluation of the S-PIV images is performed using the TSI InSight4G software. The workflow comprises image pre-processing to subtract the background from all the individual images, two-pass image cross-correlation and finally post-processing and vector validation. Third order image dewarping polynomials were generated based on the calibration target. A recursive Nyquist grid was used as a grid engine for the cross-correlation. The cross correlation engine was a Fast Fourier Transform correlator. The first cross-correlation pass was made with $64 \mathrm{px} \mathrm{x}$ $64 \mathrm{px}$ interrogation areas. A $5 \mathrm{px}$ x $5 \mathrm{px}$ vector validation was applied before the second cross-correlation pass on 32 px x $32 \mathrm{px}$ interrogation areas. The window overlap was set to $50 \%$ which yielded a $1.2 \mathrm{~mm}$ x $1.2 \mathrm{~mm}$ spatial resolution after the second pass. The signal to noise filter was set to 1.8. The number of spurious vectors after the second pass validation and smoothing process was under $10 \%$ of the overall velocity vectors. After the final vector smoothing pass a flow field comprising approximately 9,000 velocity vectors is obtained at the AIP which corresponds to a resolution of $0.8 \%$ of the AIP diameter in both $\mathrm{x}$ and $\mathrm{y}$ direction.

The reconstruction of the velocity vectors from the two off-axis recordings contain potential errors that originate from the misalignment between the calibration target and the laser light sheet (image mis-registration errors). This misalignment and its correction is often referred to as disparity correction and is explained in detail by Raffel et a $1^{14}$. Within the current study these errors were accounted for by calculating the disparity maps and correcting the calibration polynomials accordingly. The disparity processing was iteratively done in multiple passes using the entire dataset of images on order to achieve the best correction possible. The mean mis-registration error after multiple disparity corrections was typically around $3 \mathrm{px}$ except for the regions very close to the walls where the misregistration was estimated up to 5 px due to local laser light scattering effects. The analysis of Raffel ${ }^{14}$ was followed to provide an estimate of the overall S-PIV uncertainties. This analysis takes into account the particle image displacement, particle image diameter, the seeding density the quantisation level and the background noise. Overall the uncertainty on the measured in-plane velocity was approximately $8 \%$ and $4 \%$ on the out of plane velocity. 


\section{Results and discussion}

\section{A. Time averaged and unsteady flow field analysis}

The test matrix of the flow measurements is shown in Table 1 where the S-duct inlet Mach number ranged from 0.27 to 0.60 and the Reynolds number $\left(\operatorname{Re}_{\mathrm{D}}\right)$ ranged from $5.9 \times 10^{5}$ to $13.8 \times 10^{5}$. For the calculation of the inlet Mach number, the Mach number profile measured at the inlet reference plane was an area weighted average to account for the boundary layer regions. Using the Mach number profile at $0.9 \mathrm{D}_{\mathrm{i}}$ and the inlet total temperature the velocity profile was determined and the incoming compressible boundary layer displacement thickness, momentum thickness and shape factor were calculated for the two configurations across the range of inlet Mach number between 0.27 0.6. The inlet boundary layer upstream of the S-duct inlet had a thickness ranging from $3.8 \mathrm{~mm}$ to $6.7 \mathrm{~mm}$ across the Mach number range. The inlet Mach number was found to have a modest effect on the boundary layer properties; the displacement thickness reduces from approximately $0.8 \mathrm{~mm}$ to $0.7 \mathrm{~mm}$ between inlet Mach 0.27 and 0.6 in both configurations. The concomitant shape factor ranged between 1.6 to 1.8 and it was assumed the incoming boundary layer is fully turbulent.

The time averaged flow fields and statistical characteristics were calculated using 1000 flow snapshots which were acquired at $7.5 \mathrm{~Hz}$. It is of interest to understand for this dynamic flow field if this is a sufficient dataset. Fig. 5a shows the time averaged out of plane velocity, $\mathrm{w}$, and its fluctuations, $\sigma_{\mathrm{w}}$, for datasets of 250, 500, 750 and 1000 PIV snapshots for the low offset S-duct at inlet Mach $=0.27$. The contours are non-dimensionalised by a reference out of plane velocity $\mathrm{W}_{\text {ref }}=\frac{1}{A} \int_{A}\langle w(r, \theta)\rangle d A$ which is the area-averaged, time-averaged out of plane velocity at the AIP. The impact of number of snapshots on the area averaged out of plane velocity $\mathrm{W}$ and its area averaged fluctuations $\sigma_{\mathrm{W}}$ is shown in Fig. 5b. It can be seen that for 1000 snapshots the characteristics converge within less than $0.05 \%$ for the average velocity and within $2 \%$ for its rms values, hence 1000 instantaneous images are considered to be adequate. Similar convergence studies were conducted for the high offset case (Duct 2) and the adequacy of 1000 images per dataset was established as the characteristics for the out of plane velocity converged within $0.11 \%$ and $2.3 \%$ for its rms values.

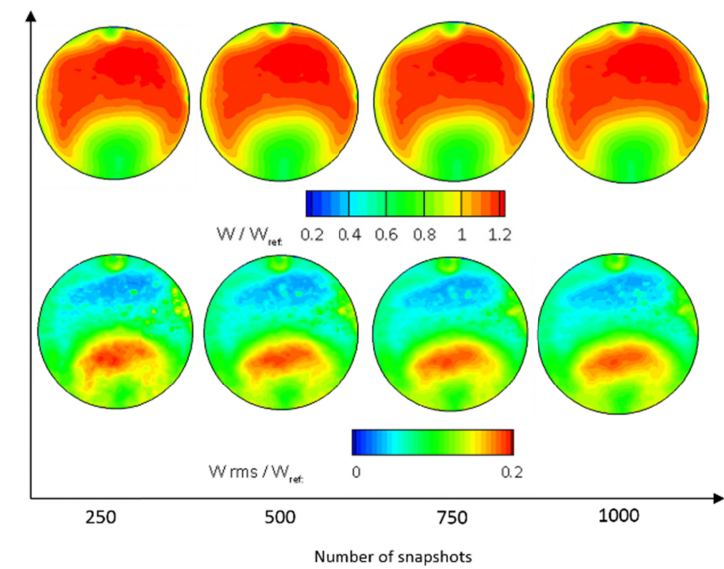

a)

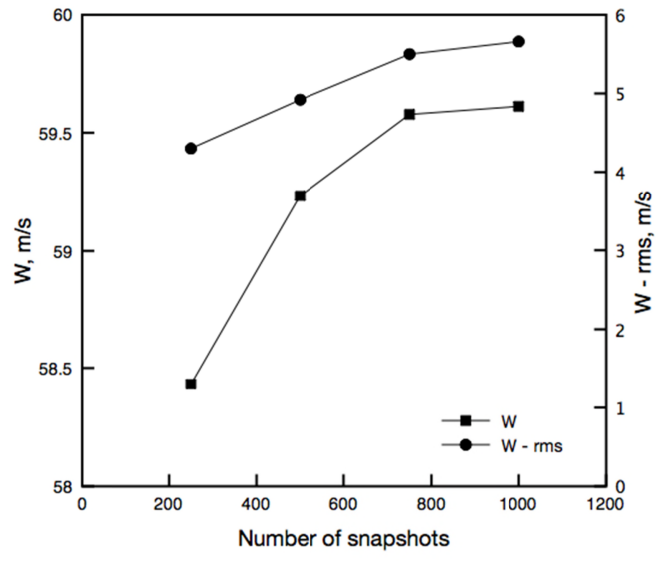

b)

Figure 5. Effect of number of snapshots on spatial distributions (a) and area averaged (b) $W$ and $\sigma_{W}$ for Duct $1\left(\mathrm{H} / \mathrm{D}_{\mathrm{i}}=\mathbf{1 . 3 4}\right)$ at inlet Mach 0.27 .

For the test configurations considered, time-averaged values of non-dimensional out of plane velocity, out of plane vorticity and swirl angle are calculated at the AIP and presented for the lowest and highest inlet Mach number cases. The anticipated low velocity zone appears in both configurations as shown in Fig. 6. In accordance with previous studies ${ }^{3-5}$ it is more pronounced in the high offset configuration $\left(\mathrm{H} / \mathrm{D}_{\mathrm{i}}=2.44\right)$ where it extends to approximately $50 \%$ of the AIP area, while it covers around $30 \%$ of the area for the low offset Duct $1\left(\mathrm{H} / \mathrm{D}_{\mathrm{i}}=1.34\right)$. The increase in the extent of the low velocity region is related to both the stronger secondary flows which arise in the high offset configuration as well as the expected upstream movement of the centreline separation point. In addition, these stronger secondary flows for the more aggressive Duct $2\left(\mathrm{H} / \mathrm{D}_{\mathrm{i}}=2.44\right)$ also result in a radial migration of the main loss region to a more central location AIP. This is of note as it is known that the conventional 
Table 1. Test matrix for S-PIV tests.

\begin{tabular}{ccccc}
\hline \hline Case no. & Duct type & Inlet Re $\mathrm{D}_{\mathrm{D}}$ & $\begin{array}{c}\text { Area averaged } \\
\text { inlet Mach }\end{array}$ & $\mathrm{W}_{\text {ref }}[\mathrm{m} / \mathrm{s}]$ \\
\hline 1 & Duct 1 & $5.9 \times 10^{5}$ & 0.27 & 58.2 \\
2 & $\mathrm{H} / \mathrm{D}_{\mathrm{i}}=1.34$ & $9.9 \times 10^{5}$ & 0.45 & 93.3 \\
3 & $\mathrm{~L} \mathrm{D}_{\mathrm{i}}=5.0$ & $13.2 \times 10^{5}$ & 0.6 & 118.0 \\
\hline 4 & Duct 2 & $6.01 \times 10^{5}$ & 0.27 & 61.5 \\
5 & $\mathrm{H} / \mathrm{D}_{\mathrm{i}}=2.44$ & $10.05 \times 10^{5}$ & 0.45 & 91.2 \\
6 & $\mathrm{~L} / \mathrm{D}_{\mathrm{i}}=5.0$ & $13.8 \times 10^{5}$ & 0.6 & 119.3 \\
\hline \hline
\end{tabular}

flow distortion descriptors, and the compression systems, are sensitive to the radial position of the flow distortions ${ }^{6,8}$. In addition, previous measurements of the area-averaged total pressure distributions at the AIP showed that the high offset configuration had a greater pressure loss and that the pressure recovery was 0.952 relative to a better performance of pressure recovery 0.971 for the low offset Duct $1^{4,5}$. Finally, the effect of the inlet Mach number, and the associated changes in Reynolds number across the range of 0.6 to $1.4 \times 10^{6}$, does not have a strong effect on the out of plane velocity distributions. There is a modest noticeable effect whereby the circumferential extent of the low velocity region is slightly reduced when the Mach number is increased from 0.27 to 0.6 (Fig. 6). This may be associated with the increase in Reynolds number and the concomitant reduction in the magnitude of the boundary layers which form into the secondary flow loss regions.

Major differences in the unsteadiness characteristics are observed between the two geometries when the nondimensional offset is $\mathrm{H} / \mathrm{D}_{\mathrm{i}}$ is increased from 1.34 to 2.44 (Fig. 7, Fig. 8, Fig. 9). For the low offset configuration (Duct $1 \mathrm{H} / \mathrm{D}_{\mathrm{i}}=1.34$ ) the out of plane velocity fluctuation distribution $\sigma_{\mathrm{w}}$ shows a local maximum of about $18 \%$ (Fig. 7) which is positioned at the top of the loss region highlighted in the time averaged w distributions (Fig. 6). It is also notable that the unsteadiness is reduced to about $10 \%$ in the main loss region close to the wall. In the rest of the AIP plane the $\sigma_{\mathrm{w}}$ levels are relatively low and below $5 \%$. There is also a local maximum of $\sigma_{\mathrm{w}}=10 \%$ at the top of the AIP plane which is associated with the minor local loss in this region. As the Mach number increases from 0.27 to 0.60 , the general distribution of the maximum $\sigma_{\mathrm{w}}$ is still concentrated in the region at the top of the loss zone and the peak values have increased slightly to about $20 \%$ (Fig. 7). The other $\sigma_{\mathrm{w}}$ local maximum at the top of the duct is similar and the local minimum at the bottom of the duct is broadly unaffected by the change in Mach numbers. There is a notable change in the unsteady $\sigma_{\mathrm{w}}$ fluctuation for the high offset configuration (Duct $2 \mathrm{H} / \mathrm{D}_{\mathrm{i}}=2.44$ ) (Fig. 7). The clearest change is that the region of peak unsteadiness is notably more extensive, with greater peak values $(20 \%)$ and located in a more central position. However, it is similar to the $\mathrm{H} / \mathrm{D}_{\mathrm{i}}=1.34$ configuration in that the main region of $\sigma_{\mathrm{w}}$ is also associated with the upper extent of the main loss zone. The radial position of the unsteadiness is notable as the impact on downstream turbomachinery depends on the level of unsteadiness as well as the radial (or spanwise) position. Just like the Duct 1 measurements, the high offset Duct 2 also exhibits a notable local maximum of $\sigma_{\mathrm{w}}$ at the top of the AIP with relatively high values of about $18 \%$. Finally, the other characteristic associated with the high offset Duct 2, is that there is no longer an extensive region of substantially low $\sigma_{\mathrm{w}}$ and that the levels are generally higher across the AIP. An increase in Mach number from 0.27 to 0.6 does not notably change the topology of $\sigma_{\mathrm{w}}$ for Duct 2. There is just a broad increase in the overall levels and the maximum $\sigma_{\mathrm{w}}$ increases to over 20\% (Fig. 7). 

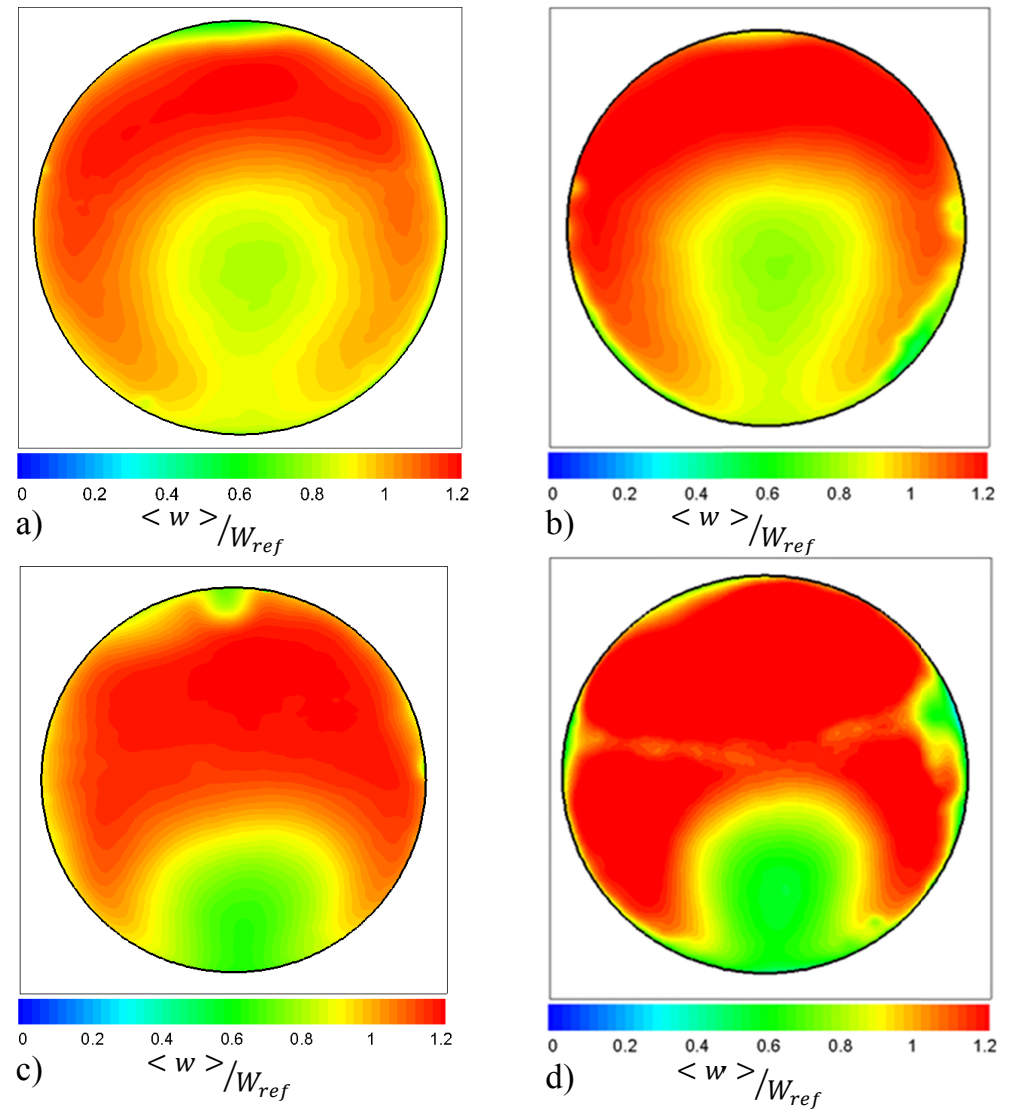

Figure 6. Time averaged out of plane velocity $\left\langle w>/ w_{\text {ref }}\right.$ at the AIP. (a) $\mathrm{H} / \mathrm{D}_{\mathrm{i}}=2.44$, inlet $\mathrm{Mach}=0.27$, (b) $\mathrm{H} / \mathrm{D}_{\mathrm{i}}=\mathbf{2 . 4 4}$, inlet Mach $=0.6$, (c) $\mathrm{H} / \mathrm{D}_{\mathrm{i}}=1.34$, inlet Mach $=0.27$, (d) $\mathrm{H} / \mathrm{D}_{\mathrm{i}}=1.34$, inlet $\mathrm{Mach}=0.6$.

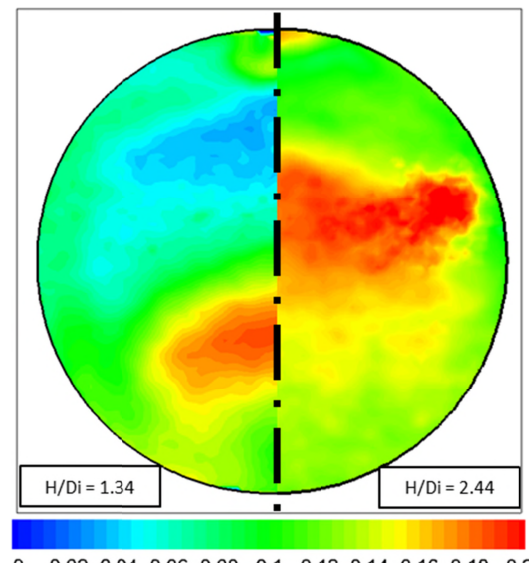

$\begin{array}{lllllllllll}0 & 0.02 & 0.04 & 0.06 & 0.08 & 0.1 & 0.12 & 0.14 & 0.16 & 0.18 & 0.2\end{array}$

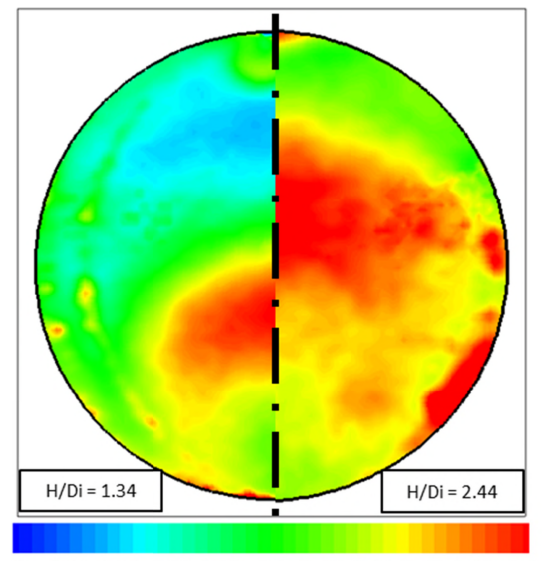

$\begin{array}{lllllllllll}0 & 0.02 & 0.04 & 0.06 & 0.08 & 0.1 & 0.12 & 0.14 & 0.16 & 0.18 & 0.2\end{array}$

b)

$$
\sigma_{w} / W_{\text {ref }}
$$

Figure 7. Out of plane velocity $\sigma_{w} / W_{\text {ref }}$ fluctuations at the AIP. $H / \mathbf{D}_{\mathbf{i}}=1.34$, inlet Mach $=0.27$ (a-left), $H / D_{i}=2.44$, inlet Mach = 0.27 (a-right), H/D $D_{i}=1.34$, inlet Mach = 0.6 (b-left), $H / D_{i}=2.44$, inlet Mach $=0.6$ (b-right). 

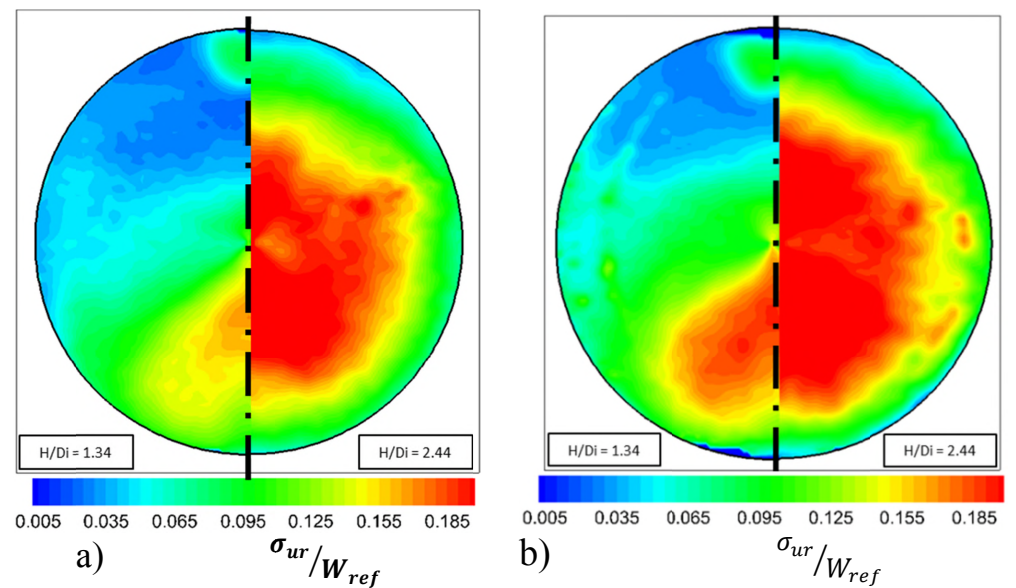

Figure 8. Radial velocity $\sigma_{u r} / W_{\text {ref }}$ fluctuations at the AIP. $\mathrm{H} / \mathrm{D}_{\mathrm{i}}=1.34$, inlet $\mathrm{Mach}=0.27$ (a-left), $\mathrm{H} / \mathrm{D}_{\mathrm{i}}=$ 2.44, inlet Mach $=0.27$ (a-right), H/D $\mathrm{D}_{\mathrm{i}}=1.34$, inlet $\mathrm{Mach}=0.6\left(\mathrm{~b}\right.$-left), $\mathrm{H} / \mathrm{D}_{\mathrm{i}}=\mathbf{2 . 4 4}$, inlet $\mathrm{Mach}=\mathbf{0 . 6}(\mathrm{b}-$ right).
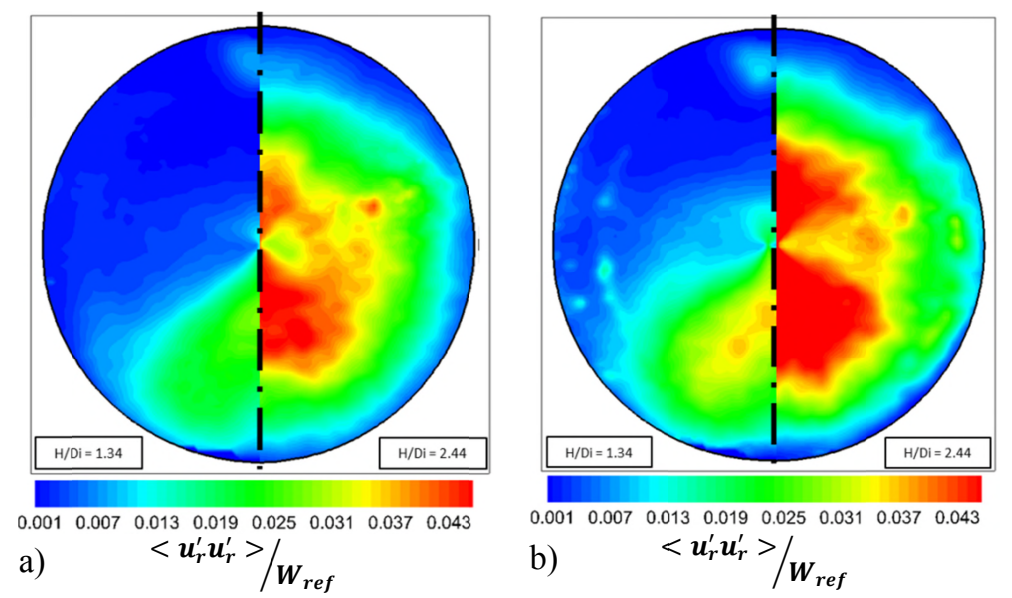

Figure 9. Time averaged $<u_{r}^{\prime} u_{r}^{\prime}>/ W_{r e f}^{2}$ Reynolds stress distribution at the AIP. H/D $\mathbf{D}_{\mathrm{i}}=1.34$, inlet Mach $=$ 0.27 (a-left), $H / D_{i}=2.44$, inlet Mach $=0.27$ (a-right), $H / D_{i}=1.34$, inlet Mach $=0.6$ (b-left), $H / D_{i}=2.44$, inlet Mach $=0.6$ (b-right).

The unsteady radial and circumferential velocity fluctuations $\left(\sigma_{\mathrm{ur}}\right.$ and $\left.\sigma_{\mathrm{u} \theta}\right)$ shown in Fig. 8 and Fig. 10 exhibit a notably different characteristic in comparison with the $\sigma_{\mathrm{w}}$ distributions (Fig. 7). At the low offset configuration the highly unsteady zone of radial velocity is located at the lower part of the AIP where the out of plane velocity is also shown to be highly unsteady. The unsteady radial velocity for the high offset case demonstrates two distinct regions in the upper and lower quadrants and the levels are around 10\% higher and located closer to the center of the AIP. Inlet Mach number does not have a notable impact on the spatial distributions except that the region of relatively high unsteadiness is almost doubled for both cases. The different behavior of the high offset configuration demonstrates some evidence of a vertical oscillatory flow centered around the mid-point of the AIP plane. This argument is also supported by the $u_{\mathrm{r}}{ }^{\prime} u_{\mathrm{r}}$ ' Reynolds stress distribution which is shown in Fig. 9. For the low offset configuration up to $3 \%$ higher flow oscillations are observed within the lower half of the AIP than within the rest of the flow field. This structure is notably different at the high offset $S$-duct. $\mathrm{u}_{\mathrm{r}}{ }^{\prime} \mathrm{u}_{\mathrm{r}}{ }^{\prime}$ increases its maximum value to 
around $4.5 \%$ and is distributed symmetrically across the $\mathrm{x}$ axis of the AIP. This suggests that the oscillatory flow patterns extend across both the upper as well the lower part of the AIP. It is anticipated that this is associated with the shedding of the separated flow from in within the duct, which in the high offset case manifests as a more intense vertical oscillatory pattern at the AIP. In addition, the inlet Mach number does not affect the general flow topology for either the low- or high-offset configurations but just increases the unsteadiness and extent of the relatively unsteady regions.

For the unsteady circumferential velocity distributions for Duct 1 at low Mach number (Fig. 10a-left), the maximum $\sigma_{\mathrm{u} \theta}$ is located close to the center of the loss region and at a greater radial position relative to the $\sigma_{\mathrm{w}}$ maximum. The maximum is about $14 \%$ which decreases to values less than $5 \%$ in the other main regions of the AIP. As with the $\sigma_{\mathrm{w}}$, the unsteadiness associated with the loss region at the top of the AIP is also in evidence with a level of $\sigma_{\mathrm{u} \theta}$ of about $9 \%$. This is similar to the effect on the out of plane velocity unsteadiness $\left(\sigma_{\mathrm{w}}\right)$. For Duct 1 when the Mach number is increased from 0.27 to 0.60 , the distribution of $\sigma_{\mathrm{u} \theta}$ is unaffected but the levels increase to about $17 \%$ with very high values also arising at the outer edge of the AIP (Fig. 10b-left). For Duct 2 the effect of the increased $\mathrm{H} / \mathrm{D}_{\mathrm{i}}$ on the $\sigma_{\mathrm{u} \theta}$ is relatively modest. The distribution is mostly the same as for Duct 1 with just a slightly greater extent and a more notable increase in the peak values from $14 \%$ to $20 \%$ (Fig. 10a-right). This is in marked contrast with the impact on the $\sigma_{\mathrm{w}}$ distribution where the characteristic distributions changed more substantially. Finally, and in agreement with the other characteristics, for Duct 2 the increase in Mach number does not alter the distribution of $\sigma_{\mathrm{u} \theta}$ but the peak values increase from about $20 \%$ to $22 \%$ (Fig. 10b-right).

The swirl angle $\alpha$ is defined by the angle between the circumferential velocity $\mathrm{u}_{\theta}$ and the axial velocity $\mathrm{w}^{6}$. The time averaged swirl angle distributions are shown in Fig. 11 for both configurations as well as for Mach 0.27 and 0.60 . Some notable differences can be observed between the two cases. The high offset configuration features approximately $40 \%$ of the AIP at large values of absolute swirl angle of around $8-10^{\circ}$. High swirl regions are more localized in the low offset configuration and mostly restricted within the lower part of the AIP. The distributions of the unsteady swirl angle are shown in Fig. 12 and for both cases it is significant that the local $\sigma_{\alpha}$ levels are over $15^{\circ}$ which is, in general, greater than the time averaged levels. Clearly there is a very significant level of unsteady swirl arising for both geometries across all Mach numbers. Duct $1\left(\mathrm{H} / \mathrm{D}_{\mathrm{i}}=1.34\right)$ shows a more localized unsteady swirl angle region located at the lower part of the AIP. This is dictated by the location of the maximum unsteadiness of $\mathrm{u}_{\theta}$ which dominates the effect relative to the variations in w (Fig. 7 and Fig. 10 respectively). The high offset configuration shows a much larger region of unsteady swirl angles and in this case it is aligned with regions of high $\sigma_{\mathrm{w}}$, in the center, and $\sigma_{\mathrm{u} \theta}$ which extends from the lower part to the centre. The indication is that for this high offset geometry, both $\sigma_{\mathrm{u} \theta}$ and $\sigma_{\mathrm{w}}$ contribute equally to the unsteadiness distribution of the swirl angle $\alpha$. Inlet Mach number has only a small effect on the swirl unsteadiness as the peak values increases by approximately 2 degrees between Mach $0.27-0.6$ in both cases. The topology of the swirl rms distribution, however, remains broadly unchanged.
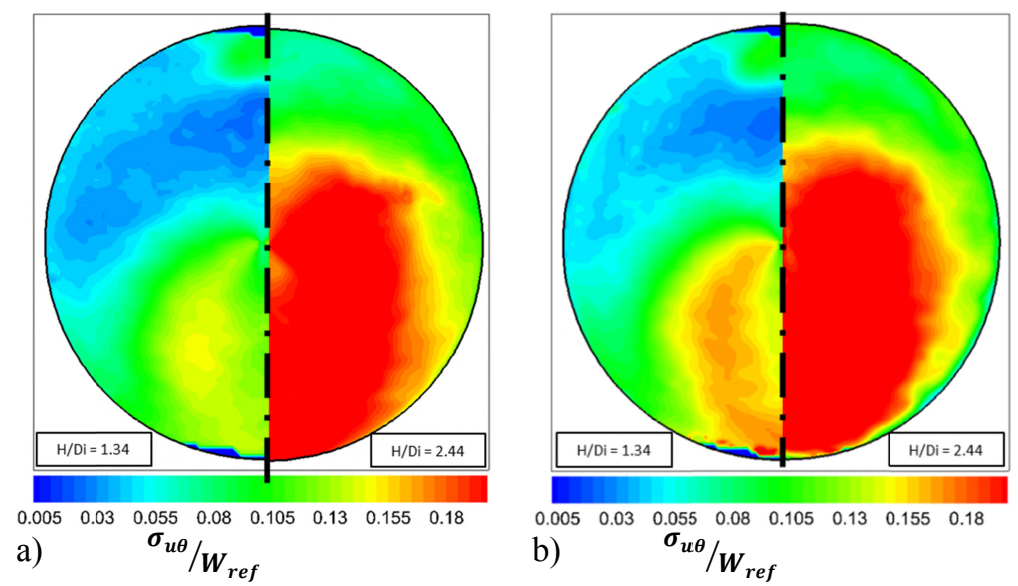

\footnotetext{
Figure 10. Circumferential velocity $\sigma_{u \theta} / W_{\text {ref }}$ fluctuations at the AIP. $\mathrm{H} / \mathrm{D}_{\mathrm{i}}=1.34$, inlet Mach $=0.27$ (aleft), $\mathrm{H} / \mathrm{D}_{\mathrm{i}}=\mathbf{2 . 4 4}$, inlet Mach $=0.27$ (a-right), $\mathrm{H} / \mathrm{D}_{\mathrm{i}}=1.34$, inlet Mach $=0.6$ (b-left), $\mathrm{H} / \mathrm{D}_{\mathrm{i}}=\mathbf{2 . 4 4}$, inlet Mach $=0.6$ (b-right).
} 


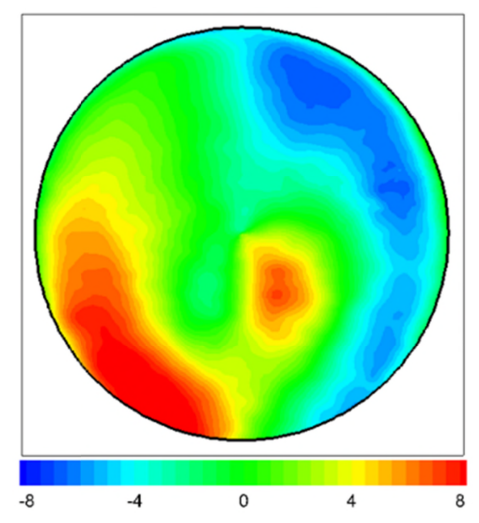

a)

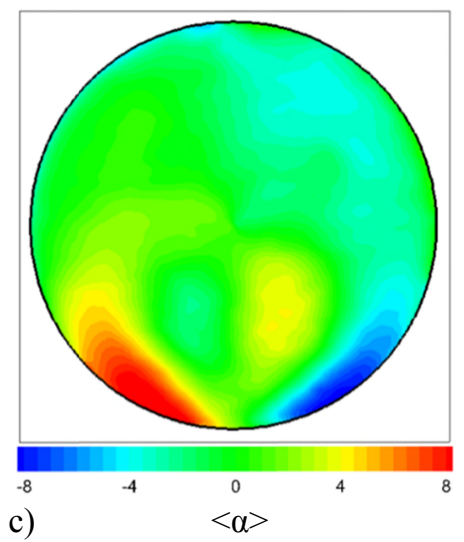

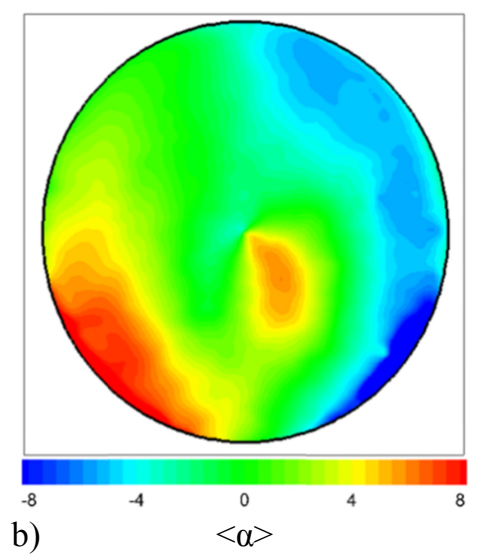

b)

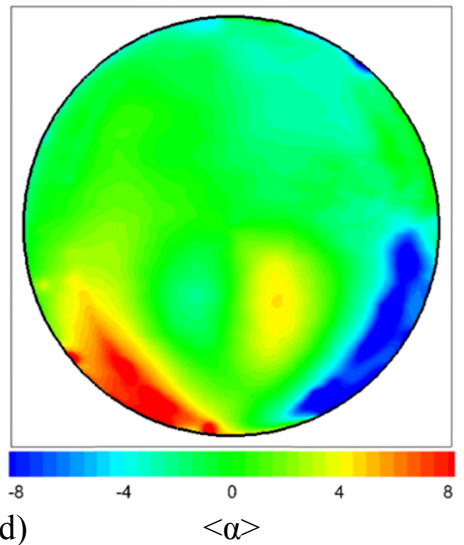

Figure 11. Time averaged $<\alpha>$ swirl angle distributions at the AIP. (a) $H / D_{i}=2.44$, inlet $M a c h=0.27$, (b), $\mathrm{H} / \mathrm{D}_{\mathrm{i}}=\mathbf{2 . 4 4}$, inlet Mach $=0.6,(\mathrm{c}), \mathrm{H} / \mathrm{D}_{\mathrm{i}}=1.34$, inlet Mach $=0.27$, (d) $\mathrm{H} / \mathrm{D}_{\mathrm{i}}=1.34$, inlet $\mathrm{Mach}=0.6$. 

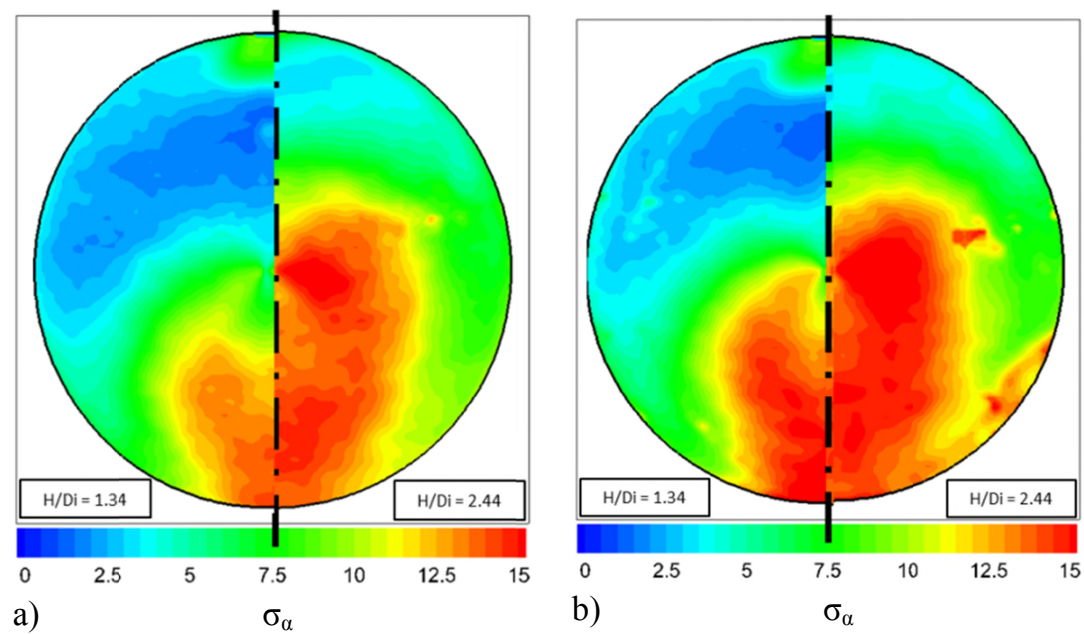

Figure 12. Swirl angle $\sigma_{a}$ fluctuations at the AIP. $H / D_{\mathrm{i}}=1.34$, inlet $\mathrm{Mach}=0.27$ (a-left), $\mathrm{H} / \mathrm{D}_{\mathrm{i}}=\mathbf{2 . 4 4}$, inlet Mach $=0.27$ (a-right), H/D $\mathrm{D}_{\mathrm{i}}=1.34$, inlet Mach $=0.6$ (b-left), $\mathrm{H} / \mathrm{D}_{\mathrm{i}}=\mathbf{2 . 4 4}$, inlet Mach $=0.6(\mathrm{~b}$-right).

To illustrate the dynamic nature of the flow and the instantaneous distributions of flow velocities and swirl angles the spatial distribution of out of plane velocity and swirl angle, at three random time instances are shown in Fig. 13 for Duct 2 at inlet Mach $=0.27$. It is of worth noting the substantial variations of the flow in each snapshot compared to the time averaged distribution. It can be therefore argued that assessments based only on time averaged measurements may not suffice for the characterization of such flows. Furthermore the very substantial changes in swirl angle and flow topology highlight that AIP assessments based on a typical 40 probe pressure, or even unsteady pressure, seem insufficient to capture the complex nature of the flow.
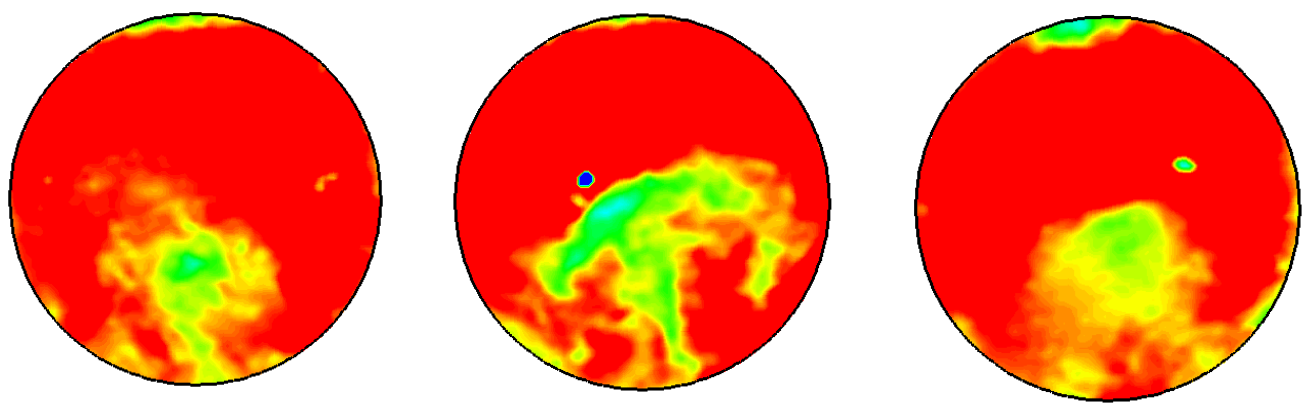

$w / W_{\text {ref }}$
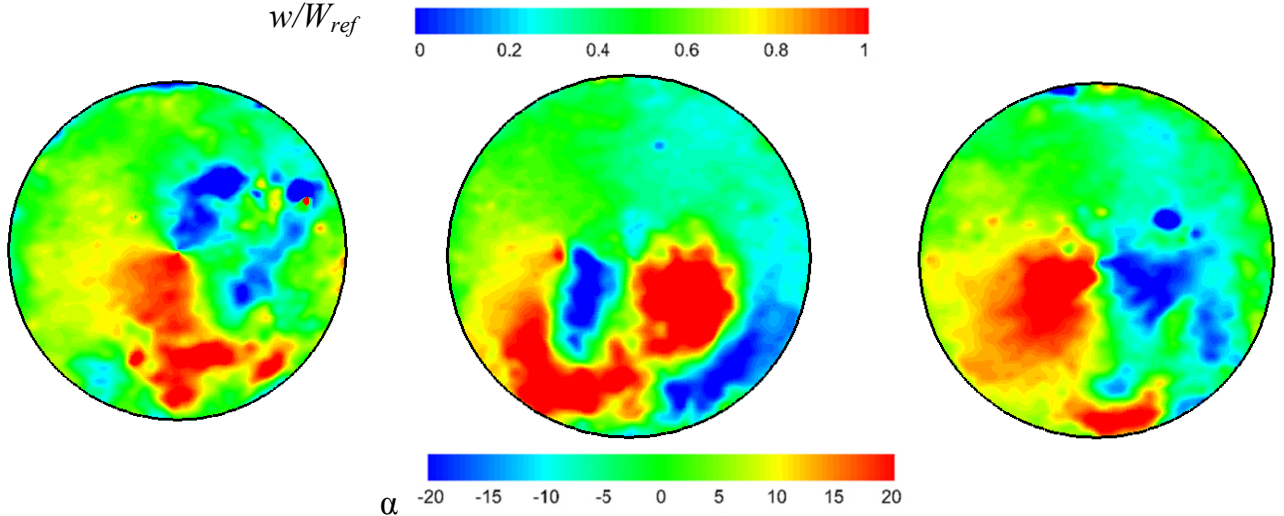

Figure 13. Instantaneous axial velocity $w / w_{\text {ref }}$ (top) and swirl angle $\alpha$ (bottom) distributions at the AIP.

High offset S-duct at inlet Mach $=0.27$. 
Finally, the distributions of the out of plane vorticity, $\left(\omega_{z}=\frac{\partial v}{\partial x}-\frac{\partial u}{\partial y}\right)$, are presented in Fig. 14 for both configurations. As anticipated the highest vorticity magnitude presents itself at the lower half of the AIP where the vortical flows also observed by Wellborn et $\mathrm{al}^{3}$ and Garnier et $\mathrm{al}^{5}$ are located. The peak vorticity at the high offset configuration $\left(H / D_{i}=2.44\right)$ is around $20-30 \%$ higher than in the low offset case $\left(H / D_{i}=1.34\right)$. The vortical patterns are positioned closer to each other in the high offset case and slightly closer to the center of the AIP, while the low offset configuration demonstrates around 15-20\% bigger distance between the vortex centers which are also located at a lower position closer to the bottom edge of the AIP. As anticipated, the inlet Mach number seems to have a small effect in the vorticity distributions. By comparing the time averaged vorticity distributions with the turbulence intensity of the out of plane velocity component $\mathrm{w}$ shown in Fig. 7 above, it can be realized that the location of the velocity unsteadiness does not coincide with the location of the highest vorticity magnitudes as shown in Fig. 14. This suggests that the out of plane vorticity may not be necessarily associated with the flow unsteadiness which seems to be mostly driven by the streamwise flow separation which arises along the centreline.

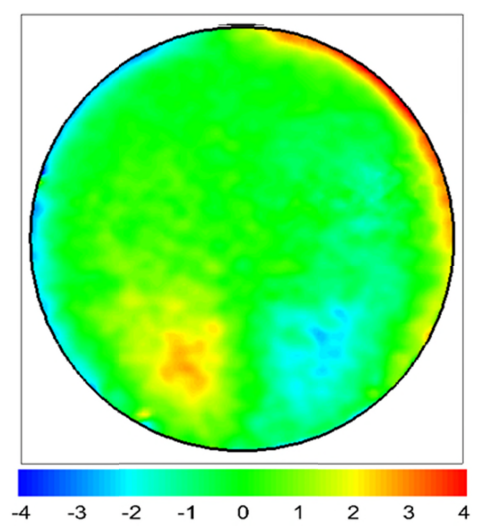

a)

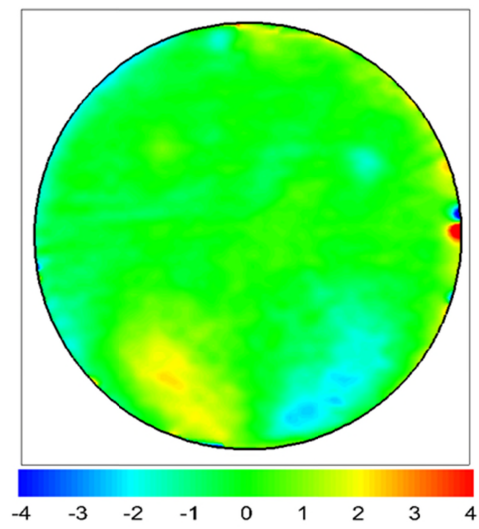

c) $\quad \frac{<\omega_{z}>D_{\text {out }}}{W_{\text {ref }}}$

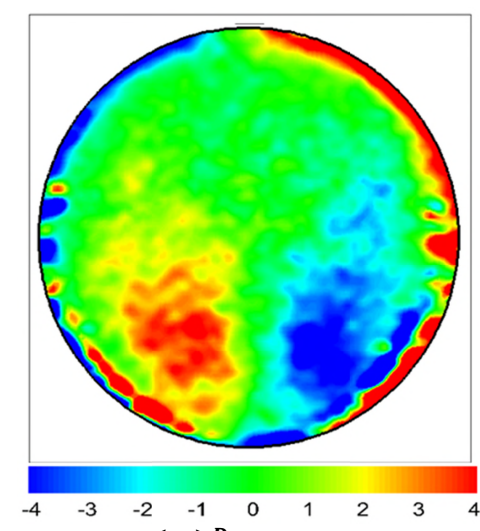

b)

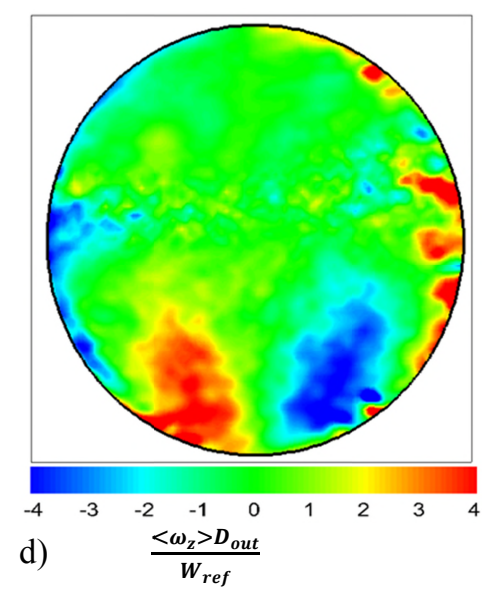

Figure 14. Time averaged vorticity $\frac{<\omega_{z}>D_{\text {out }}}{W_{\text {ref }}}$ distributions at the AIP. (a) $H / D_{i}=2.44$, inlet $M a c h=0.27$, (b) $\mathrm{H} / \mathrm{D}_{\mathrm{i}}=\mathbf{2 . 4 4}$, inlet Mach $=0.6$, (c) $\mathrm{H} / \mathrm{D}_{\mathrm{i}}=1.34$, inlet $\mathrm{Mach}=0.27$, (d) $\mathrm{H} / \mathrm{D}_{\mathrm{i}}=1.34$, inlet Mach $=0.6$. 


\section{B. Flow distortion assessment}

The S-PIV measurements provide approximately 9000 data points of the three velocity components at the AIP. As part of the industry standard on engine inlet flow distortion a range of flow distortion descriptors are proposed for swirl related distortion. For the assessment of swirl distortion the swirl angle $\mathrm{SC}(60)$ descriptor is used ${ }^{15}$ and is defined as:

$$
\operatorname{SC}(60)=\frac{\operatorname{Max}\left(u_{\theta}[60]\right)}{\mathrm{W}_{\mathrm{ref}}}
$$

where $\operatorname{Max}\left(\mathrm{u}_{\theta}[60]\right)$ is the mean value of circumferential velocity in the $60^{\circ}$ sector with the highest $\mathrm{u}_{\theta}$ average and $\mathrm{W}_{\mathrm{ref}}=\frac{1}{A} \int_{A}\langle w(r, \theta)\rangle d A$ the area averaged axial velocity at the AIP. In addition, standards have been introduced to define a set of swirl descriptors by categorizing the types of swirl distortion into four groups; bulk swirl, tightlywound vortices, paired swirl and cross flow swirl ${ }^{6}$. The definition and interpretations of these is based on a "ring and rake" approach, which is a typical experimental approach for AIP distortion measurements. The one-per-revolution, paired swirl pattern is shown in Fig. 15 and serves to illustrate the definitions of the SAE swirl distortion descriptors ${ }^{6}$.

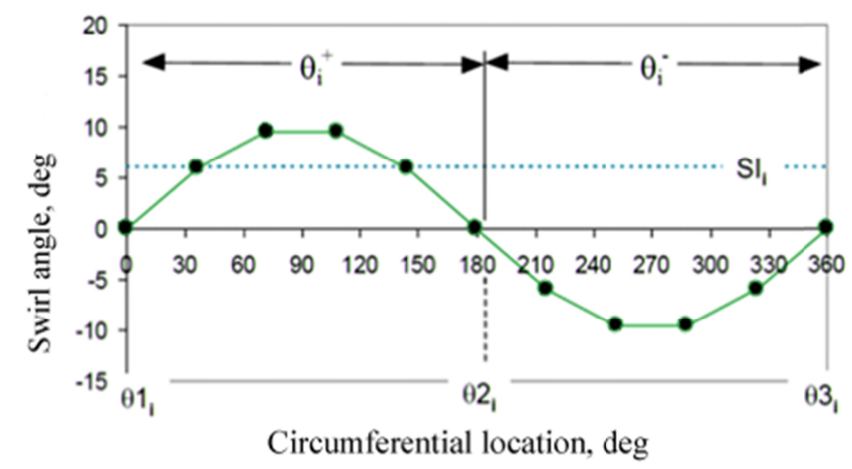

Figure 15. Typical one-per-rev symmetric paired swirl pattern for single immersion ${ }^{6}$.

Theta plus, $\theta_{i}^{+}$, is the circumferential extent of the positive (co-rotating) swirl region and theta minus, $\theta_{i}^{-}$, is the circumferential extent of the negative (counter-rotating) swirl region. The extents of these regions are defined when swirl angle changes sign. As shown above, swirl changes direction at two circumferential locations:

$$
\theta 1_{i}=0^{o}, \theta 2_{i}=180^{\circ}
$$

thus the $\theta^{+}$extent is:

$$
\theta_{i}^{+}=\theta 2_{i}-\theta 1_{i}
$$

and $\theta^{-}$extent will be:

$$
\theta_{i}^{-}=\theta 3_{i}-\theta 2_{i}
$$


Based on these definitions the SAE distortion descriptors are extended to characterize swirl of multi-per-rev patterns measured on a given ring. The swirl descriptors are defined as follows: index $i$ is used to define the ring and index $\mathrm{k}$ is used to identify the various swirl pairs along ring $i$.

Sector swirl (SS) quantifies the azimuthally averaged positive, $\mathrm{SS}_{\mathrm{i}, \mathrm{k}}^{+}$, and negative, $\mathrm{SS}_{\mathrm{i}, \mathrm{k}}^{-}$, swirl content at a given radial ring.

$$
\begin{aligned}
& S S_{i, k}^{+}=\frac{1}{\theta_{i, k}^{+}} \int_{\theta_{i, k}^{+}} \alpha(\theta)_{i, k} d \theta \\
& S S_{i, k}^{-}=\frac{1}{\theta_{i, k}^{-}} \int_{\theta_{i, k}^{-}} \alpha(\theta)_{i, k} d \theta
\end{aligned}
$$

Swirl intensity (SI) represents the average of the absolute swirl angle in degrees for a considered ring $i$. The swirl intensity across the entire AIP is given by the following expression where $\mathrm{k}$ is the number of rings considered.

$$
S I(i)=\frac{\sum_{k=1}^{m} S S_{i, k}^{+} \cdot \theta_{i, k}^{+}+\sum_{k}^{m}\left|S S_{i, k}^{-}\right| \cdot \theta_{i, k}^{-}}{360}
$$

Swirl directivity (SD) represents the overall rotational direction of the swirl with respect to the compressor rotation at each ring. In other words, this parameter provides the equivalent bulk swirl rotational direction as shown graphically in Fig. 16. It is defined as:

$$
S D(i)=\frac{\sum_{k=1}^{m} S S_{i, k}^{+} \cdot \theta_{i, k}^{+}+\sum_{k}^{m} S S_{i, k}^{-} \cdot \theta_{i, k}^{-}}{\sum_{k=1}^{m} S S_{i, k}^{+} \cdot \theta_{i, k}^{+}+\sum_{k}^{m}\left|S S_{i, k}^{-}\right| \cdot \theta_{i, k}^{-}}
$$

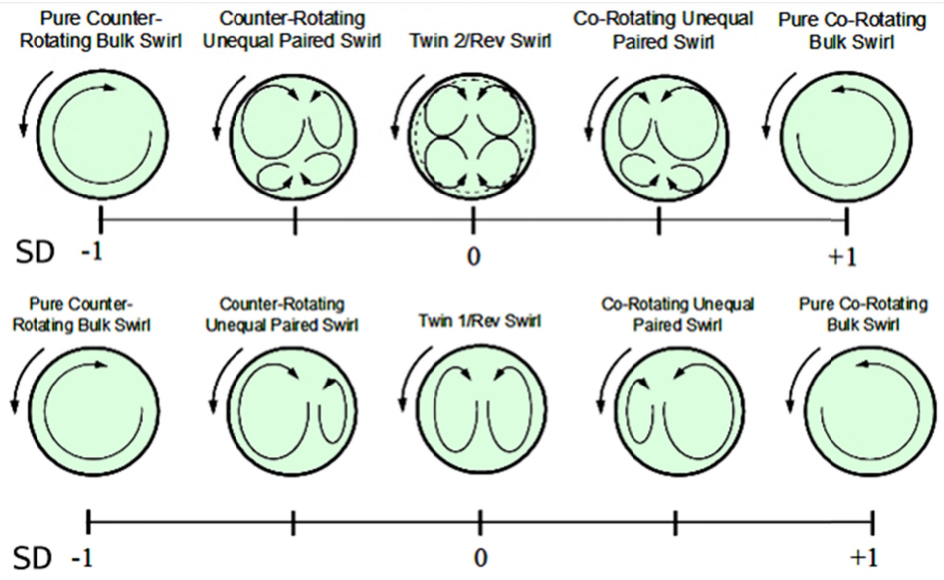

Figure 16. Swirl directivity spectrum for multiple per-rev swirl distortion compared to a one per-rev spectrum ${ }^{6}$. 
Swirl pairs (SP) indicates the number of pairs of positive and negative swirl regions present in the ring of consideration. The spectrum of swirl pairs is graphically shown in Fig. 17 and the descriptor is defined as:

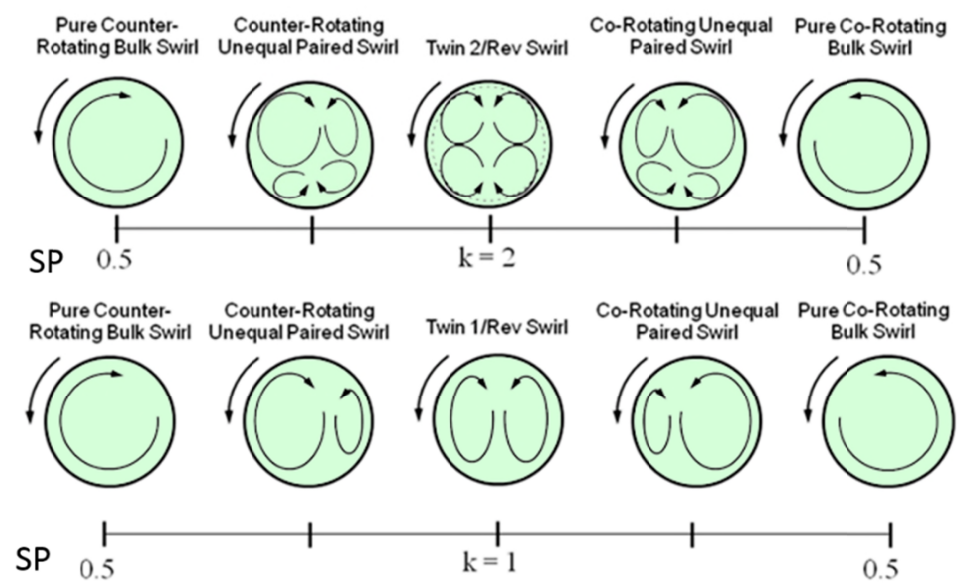

Figure 17. Swirl pairs spectrum for multiple per-rev swirl distortion compared to a one per-rev spectrum $^{6}$.

$$
S P(i)=\frac{\sum_{k=1}^{m} S S_{i, k}^{+} \cdot \theta_{i, k}^{+}+\sum_{k}^{m}\left|S S_{i, k}^{-}\right| \cdot \theta_{i, k}^{-}}{2 \cdot \operatorname{Max}\left\{S S_{i, k}^{+} \cdot \theta_{i, k}^{+},\left|S S_{i, k}^{-} \cdot \theta_{i, k}^{-}\right|\right\}_{k=1, \ldots, m}}
$$

For the calculation of the descriptor statistical properties, a descriptor value was obtained for each instantaneous flow snapshot based on a resolution of an $8 \times 5$ rake. The time averaged values were calculated based on the individual descriptors determined for each of the 1000 snapshots for each configuration. The reason that the spatial resolution was under-sampled for the calculation of the descriptors is that the industry standard and evaluation of the parameters is based on the low resolution and it was decided to be initially consistent with this approach and to simulate the characteristics of a conventional measurement approach. The time average and standard deviation of the swirl descriptors are presented in Table 2 and Fig. 18 for the considered cases. As expected, the swirl footprint of the high offset configuration is larger than that of the low offset. This can be seen by comparing the individual swirl descriptors of the two S-ducts. Swirl intensity is around $90 \%$ higher in the high offset case. This is in agreement with the swirl angle unsteadiness distribution $\sigma_{\alpha}$ shown in Fig. 12, which suggests that the extent of the highly unsteady zone for the high offset case is twice that of the low offset. The difference in SI can be evaluated also by looking at the rms distributions of the $\mathrm{w}$ and $\mathrm{u}_{\theta}$ velocity components (Fig. 7 and Fig. 10) and can be justified by the notably higher $\sigma_{\mathrm{w}}$ for the high offset configuration. Mean swirl directivity (SD) is very close to zero for the high offset case which reveals a symmetry of the swirl distortion patterns with respect to the vertical axis, while the low offset S-duct demonstrates SD levels which are very slightly biased towards negative SD values i.e. clock-wise vortex rotation. The mean swirl pairs index (SP) lies within a band of $\pm 10 \%$ from unity in both configurations which suggests that a twin one-per-rev swirl distortion pattern is present in both cases. Finally, as expected, the impact of the duct offset is highlighted in the SC60 descriptor which for the high offset Duct 2 configuration is almost double that of the low offset Duct 1 across the range of Mach numbers. Additionally, there is no strong inlet Mach number effect on of the swirl descriptors. This finding suggests that each S-duct features its own individual distortion signature that depends only on the aggressiveness of the geometry and perhaps the inlet Reynolds number which influences the levels of boundary layer based vorticity introduced into each configuration. However, further work to identify the effect of the inlet Reynolds number and the inlet boundary layer vorticity on the distortion characteristics is required. The outcome of the current study suggests a very weak dependency of the swirl descriptors on the inlet Mach number and therefore that underwrites the pertinence of tests which evaluate swirl distortion at relatively low Mach numbers. 
Table 2. Swirl descriptor and TKE statistical properties.

\begin{tabular}{ccccccc}
\hline \hline & HO_M27 & HO_M45 & HO_M60 & LO_M27 & LO_M45 & LO_M60 \\
\hline SI mean & 8.517 & 9.228 & 9.827 & 4.775 & 5.274 & 5.454 \\
SI std & 1.597 & 1.710 & 1.711 & 0.874 & 0.990 & 1.116 \\
SI max & 14.874 & 15.2227 & 15.601 & 8.893 & 10.238 & 10.685 \\
SD mean & -0.004 & 0.015 & -0.021 & -0.107 & -0.088 & -0.096 \\
SD std & 0.433 & 0.422 & 0.397 & 0.230 & 0.225 & 0.246 \\
SD max & 0.949 & 0.917 & 0.915 & 0.839 & 0.861 & 0.951 \\
SP mean & 0.899 & 0.881 & 0.898 & 1.162 & 1.147 & 1.146 \\
SP std & 0.144 & 0.143 & 0.134 & 0.170 & 0.169 & 0.173 \\
SP max & 1.473 & 1.520 & 1.427 & 1.646 & 1.861 & 1.959 \\
SC60 mean & 0.235 & 0.247 & 0.255 & 0.134 & 0.141 & 0.167 \\
SC60 std & 0.044 & 0.048 & 0.046 & 0.026 & 0.029 & 0.034 \\
SC60 max & 0.365 & 0.392 & 0.369 & 0.224 & 0.257 & 0.270 \\
TKE mean & 133.3 & 332.7 & 607.7 & 49.8 & 224.7 & 427.7 \\
TKE std & 50.4 & 124.9 & 223.4 & 19.7 & 71.8 & 139.4 \\
TKE max & 327.4 & 853.14 & 1500.5 & 169.7 & 577.2 & 1055.1 \\
\hline \hline
\end{tabular}

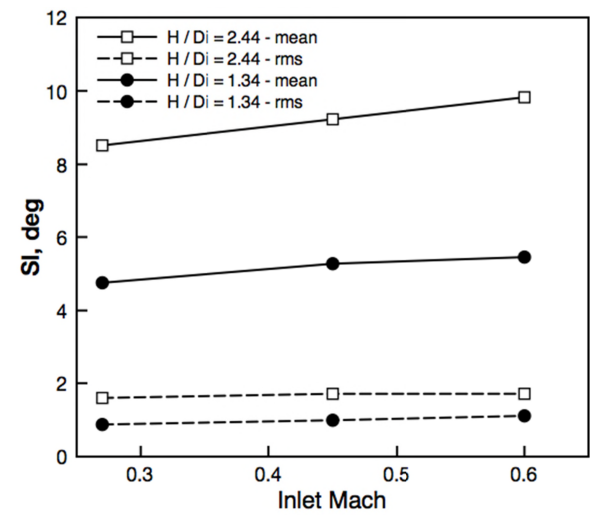

a)

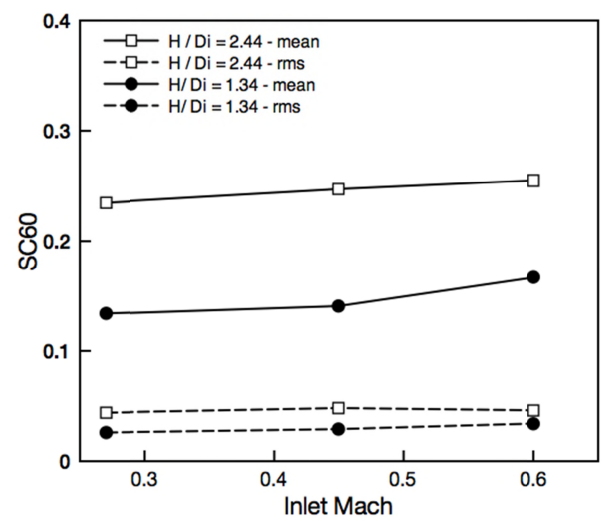

b)

Figure 18. Average and standard deviation of SI (a) and SC60 (b) for the high and low offset S-ducts across the range of inlet Mach numbers.

In addition to the mean and the variance of SI, SD, SP and SC60 the skewness, $\mathrm{s}_{\mathrm{s}}$, and the kurtosis, $\mathrm{s}_{\mathrm{k}}$, of the time signals were also obtained. The interest here is in understanding the characteristics of the unsteady variations that arise in the flow field. In particular, from the perspective of a designer, it is important to not just consider the mean and standard deviation, but also the likelihood of extreme distortion events arising is a key consideration. This is because of the highly dynamic nature of the flow and the potential sensitivity of the engine to individual events which have a global impact on the engine operation. The first step in this process, with particular emphasis on the previously neglected swirl aspects, is the evaluation of the skewness and kurtosis of the swirl descriptors. Skewness of a variable $\mathrm{x}_{\mathrm{i}}$ is defined as: 


$$
s_{S}=\frac{\frac{1}{N} \sum_{i=1}^{N}(x i-\bar{x})^{3}}{\left[\frac{1}{N} \sum_{i=1}^{N}(x i-\bar{x})^{2}\right]^{3 / 2}}
$$

Skewness measures the symmetry of a distribution with respect to the Gaussian distribution which has a skewness of zero ${ }^{16}$. Skewness becomes positive when the distribution becomes biased towards the right side of the mean and vice versa. If skewness is less than -1 or greater than +1 the distribution is considered as highly skewed. If it is between -1 and $-1 / 2$ or $+1 / 2$ and +1 the distribution is moderately skewed and when it is between $\pm 1 / 2$ the distribution is approximately symmetric. Kurtosis quantifies whether the shape of data distribution matches the Gaussian distribution ${ }^{16}$. It is defined as:

$$
s_{k}=\frac{\sum_{i=1}^{N}(x i-\bar{x})^{4}}{\left[\sum_{i=1}^{N}(x i-\bar{x})^{2}\right]^{2}}
$$

A Gaussian distribution has a kurtosis index of 3.0 (mesokurtic). A flatter distribution obtains kurtosis values lower than 3 (platykurtic), while a more peaked distribution has kurtosis higher than 3 (leptokurtic). Kurtosis is used in conjunction with skewness to assess the normality of a given distribution. A comparison between the skewness of the swirl descriptors, SI, SD, SP, SC60, which are shown in left column of Fig. 19 shows a deviation of their distribution from the Gaussian shape towards the positive direction i.e. towards the right side of the mean. Although the skewness is at a medium level it is of particular note that for the SI and SC60 parameters that the skewness is positive and therefore there is a greater likelihood of more detrimental or indeed extreme adverse events. This behavior is common between the two S-ducts tested across the entire range of inlet Mach numbers. The right hand column of Fig. 19 illustrates the kurtosis of the swirl descriptor distributions across the range of inlet Mach numbers. As can be seen, all swirl descriptors demonstrate a similarity to the Gaussian distribution as in all cases the kurtosis lies within a $\pm 15 \%$ range from the normal distribution kurtosis value of 3.0. Inlet Mach number has very little effect on the kurtosis.

Finally, an additional way to consider the distortion parameters for each configuration is shown in Fig. 20. The cloud plots illustrate the distribution of the distortion parameters of each individual S-PIV snapshot and highlight potential extreme events that may affect the stability of a downstream compression system. Fig. 20a and b show the distribution of the SC60 and SI descriptors for the two configurations at inlet Mach $=0.27$ (a) and 0.6 (b). As also demonstrated by Fig. 18, no significant changes are anticipated between the two inlet Mach numbers as each duct seems to exhibit the same distortion signature across the range of inlet Mach numbers. The correlation between SP and SD is shown in Fig. 20c and d. It is recalled that $\mathrm{SD}=0$ and $\mathrm{SP}=1$ signifies a single swirl pair of equivalent size vortices. The mean values of both parameters suggest a situation whereby SD $\sim 0$ and SP $\sim 1$ for both S-ducts across the entire range of Mach numbers. As also anticipated, any departure from SP $=1$ is associated with a simultaneous increase of SD towards \pm 1 which signifies that the swirl structures is no more a symmetric pair rather than a single dominant vortex rotating in either clock- or anti-clock wise direction. The low offset configuration has an SP range between $0.8-1.4$ with a small number of points scattered outside that zone. For the high offset case the SP range is broadly within $0.5-1.2$ with a small amount of snapshots also outside that zone. In terms of SD there seems to be a clear distribution of the majority of the individuals over the \pm 1 zone for both S-ducts. From the SD-SP maps it becomes clear that a symmetric single vortex pair dominates the AIP flowfield which is modulated to modes dominated by a single clockwise- or anti-clockwise rotating vortex. This assessment remains unaffected by the inlet Mach number. However, the SD-SP map at inlet Mach $=0.6$ shows a larger scattering of the SP zone for the low offset configurations which is approximately $0.6-1.8$ with a small number of points beyond that range but no further than $\mathrm{SP}=2$.

The effect of inlet Mach number is more clearly shown by the SI-TKE maps (Fig. 20e and f) where the turbulence kinetic energy (TKE) is defined as $\left.\frac{1}{2}\left[<u_{r}^{\prime} u_{r}^{\prime}>+<u_{\theta}^{\prime} u_{\theta}^{\prime}\right\rangle+<w^{\prime} w^{\prime}>\right]$. For the low inlet Mach number both the SI as well as the mean TKE are 50\% lower than at the high offset configuration. The positive skewness of SI provides a distinct correlation against the TKE. A similar correlation, albeit of a lower slope can be observed at inlet Mach $=0.6$, indicating that the sensitivity of the SI with TKE reduces by approximately $50 \%$ at high inlet Mach number. 
Skewness of the area averaged SI time signal

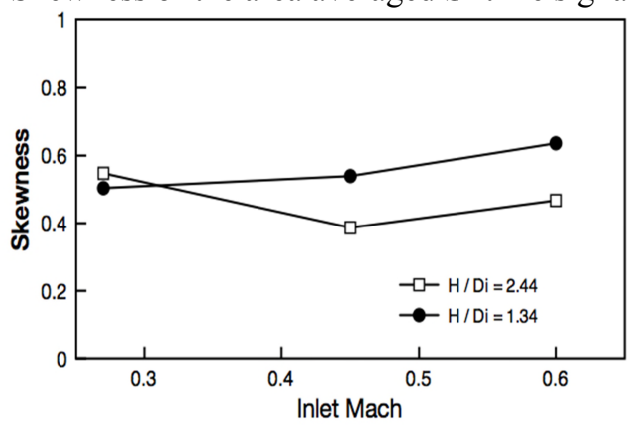

a)

Skewness of the area averaged SD time signal

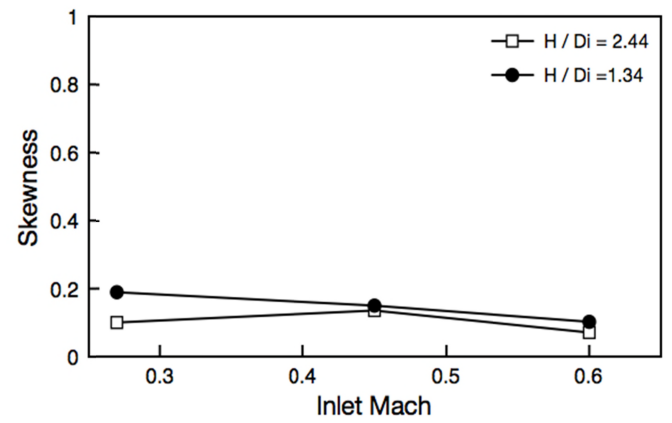

c)

Skewness of the area averaged SP time signal

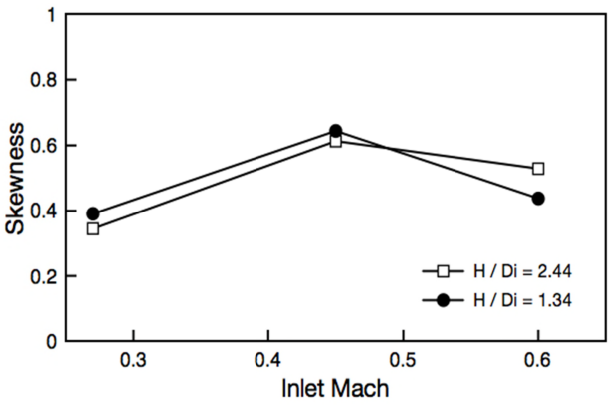

e)

Skewness of the area averaged SC60 time signal

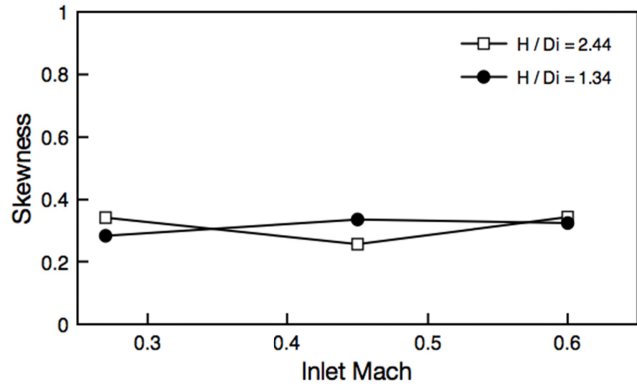

g)

Figure 19. Statistical properties of SAE distortion descriptors for the two configurations, $H / D_{i}=1.34$ and $H / D_{i}=2.44$, across the range of tested inlet Mach numbers.
Kurtosis of the area averaged SI time signal

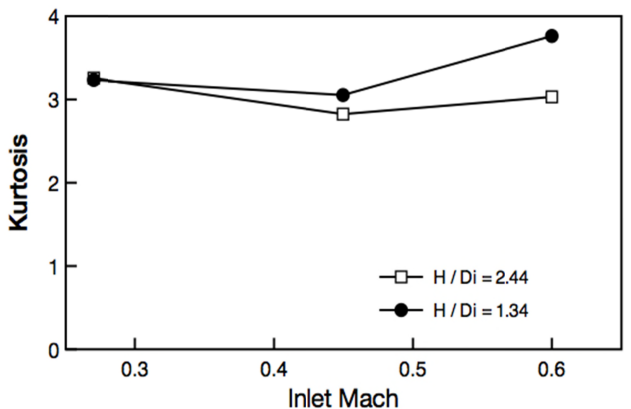

b)

Kurtosis of the area averaged SD time signal

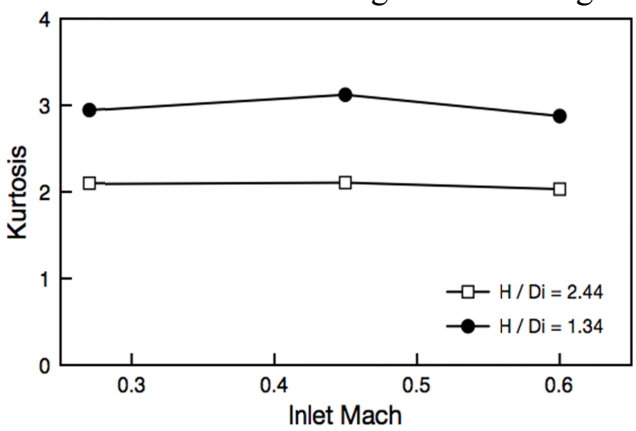

d)

Kurtosis of the area averaged SP time signal

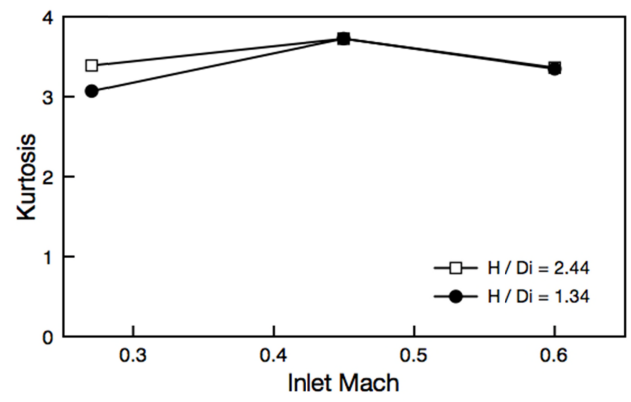

f)

Kurtosis of the area averaged SC60 time signal

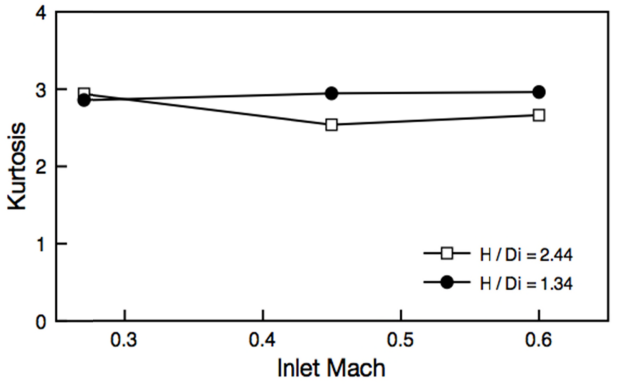

h) 


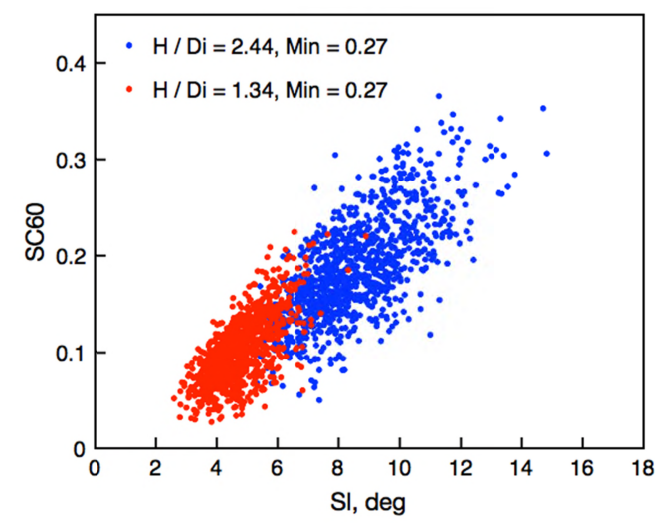

a)

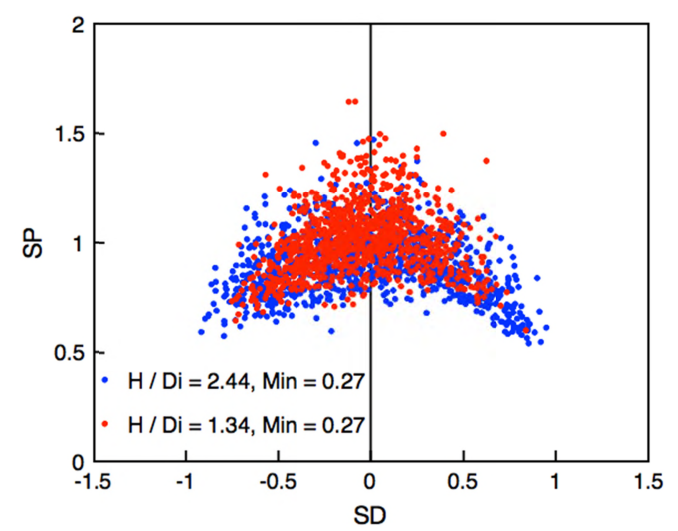

c)

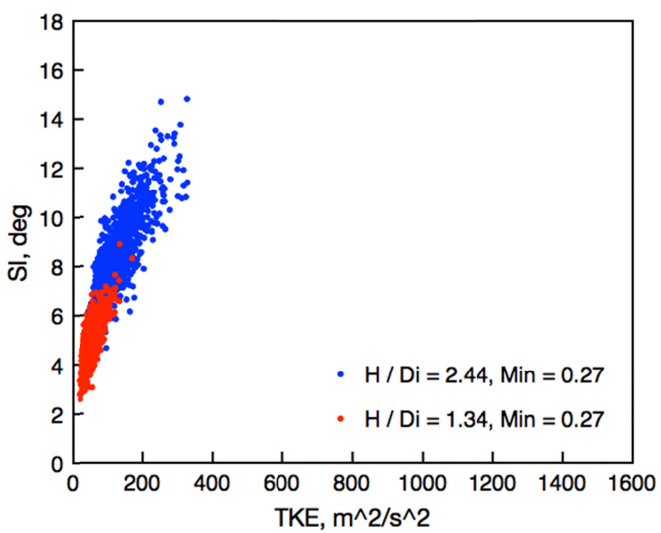

e)

Figure 20. Distortion maps for the $H / D_{i}=1.34$ and $H / D_{i}=2.44$ configuration. SC60-SI, SP-SD and SITKE maps at inlet Mach $=0.27(\mathrm{a}, \mathrm{c}, \mathrm{e})$ and at inlet $\mathrm{Mach}=0.6(\mathrm{~b}, \mathrm{~d}, \mathrm{f})$. b)

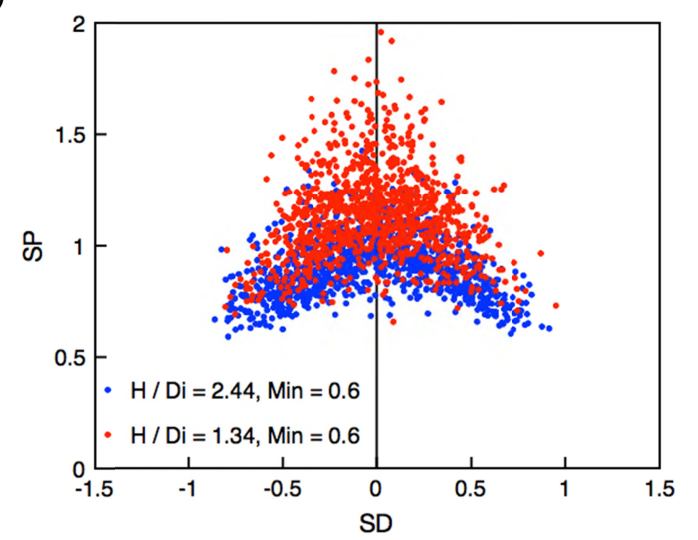

d)

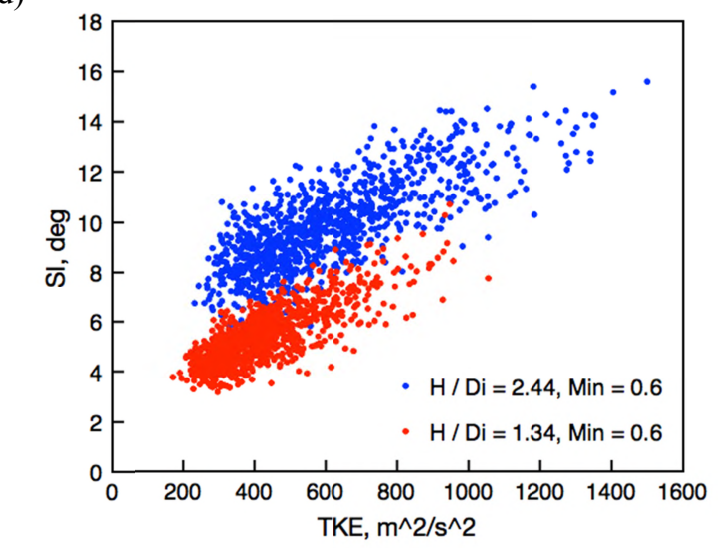

f)

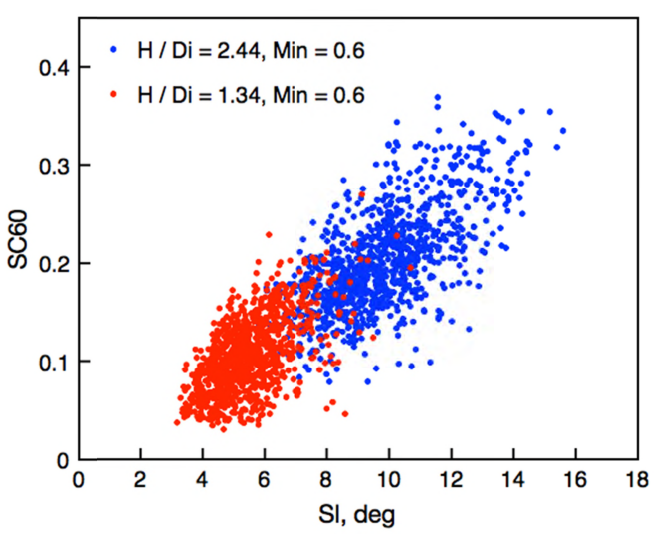

) 


\section{Conclusions}

In this study the intake flow distortions were investigated at the exit of convoluted intakes by means of stereoscopic PIV. The main challenges associated with the application of S-PIV for the measurement of the velocity fields in confined flows are addressed. The acquired velocity fields feature approximately 9,000 vectors across the entire AIP which corresponds to a resolution of $0.8 \%$ of the AIP diameter. This is significantly higher than the standard rake measurements which typically feature 40 measurement points across the AIP. The S-PIV technique allows flow analysis in terms of snapshot, statistical and time averaged measurements for two S-duct configurations with offset to inlet diameter ratios of H/Di 1.34 and 2.44 respectively across a range of inlet Mach numbers between 0.27-0.6. For the time averaged fields a convergence study showed that 1000 images per dataset suffice for a representative time averaged flow field. The time averaged fields of flow velocity, swirl angle and out of plane vorticity were compared across the AIP as well the distributions of their unsteady fluctuations and Reynolds stresses. The comparisons of the AIP flow fields revealed that the high offset configuration generates more distorted as well as more unsteady flows. The regions of high swirl angle unsteadiness are not uniquely co-located with the highly unsteady out of plane velocity part of the flow and it is postulated that these elements are related to different elements of the distorted flow. The unsteadiness of the swirl angle is more extensive for the high offset configuration and is a result of the unsteadiness that the circumferential and the out of plane velocity components demonstrate. Inlet Mach number has only a modest effect on both out of plane vorticity as well as swirl angle AIP distributions.

The conventional distortion descriptors, namely SI, SD and SP, as well as the SC60 were calculated for all the studied cases across the range of inlet Mach numbers. As expected the high offset duct of $\mathrm{H} / \mathrm{Di}=2.44$ demonstrates more distorted flows which result in higher values of SI and SC60 by around 80\% in comparison with the low offset case. The swirl descriptors show weak dependency upon the inlet Mach number for both S-ducts. The statistical analysis of the swirl properties showed that the kurtosis is similar to that of a Gaussian distribution, but that there is a positive skewness in the SI and SC60 parameters with a bias towards the more adverse distortion conditions. Distortion cloud maps allow the inspection of the swirl descriptor distributions and the identification of potential extreme events that may have an effect on the stability of a downstream compression system. The construction of a SI-SC60 map revealed a broadly linear dependency between these properties which remains unchanged across the range of inlet Mach. SD-SP cloud maps revealed the tendency of both configurations to generate a single symmetric pair of vortices as well an equal number of individuals that feature a single contra or counter rotating vortex at the AIP. Finally, SI-TKE cloud maps show a strong linear dependency of these parameters for both configurations which becomes weaker at higher Mach numbers.

In this paper the capability of S-PIV for a highly distorted, complex flow field was demonstrated whereby the substantially rich spatial resolution that S-PIV provided can be used to better understand the unsteady swirl distortion of such flow fields. The demonstrated synchronous spatial resolution is approximately 250 times greater than that provided by a typical conventional system. The key capability of the method to acquire full planar field synchronous data enables the temporal and statistical evaluation of the velocity field and swirl distortion metrics at a spatial resolution that has not been previously possible. This is a key step forward in unlocking the complex aerodynamics that dominate advanced intake-engine systems.

\section{References}

${ }^{1}$ Kawai, R., Friedman, D., Serrano, L., "Blended Wing Body Boundary Layer Ingestion Inlet Configuration and System Studies”, NASA Contract Report CR-2006-214534, 2006.

${ }^{2}$ Kim, H, and Liou, M., "Flow Simulation of N2B Hybrid Wing Body Configuration", 50th AIAA Aerospace Sciences Meeting, 2012-0838, Nashville, Tennessee, USA, 2012.

${ }^{3}$ Wellborn, S. R., Reichert, B. A., and Okiishi, T. H., "Study of the Compressible Flow in a Diffusing S-Duct", AIAA Journal of Propulsion and Power, Vol. 10, 1994, pp. 668-675.

${ }^{4}$ Delot, A.-L., Garnier, E., Pagan, D., "Flow Control in a High-Offset Subsonic Air Intake", $47^{\text {th }}$ AIAA/ASME/SAE/ASEE Joint Propulsion Conference and Exhibit, AIAA 2011-5569, San Diego, California, USA, 2011.

${ }^{5}$ Garnier, E., Leplat, M., Monnier, J., C., Delva, J., "Flow Control by Pulsed Jet in a Highly Bended S-Duct", $6^{\text {th }}$ AIAA Flow Control Conference, AIAA 2012-3250, New Orleans, Louisiana, USA, 2012. 
${ }^{6}$ S-16 Turbine Engine Inlet Flow Distortion Committee, “A Methodology for Assessing Inlet Swirl Distortion”, Report No. AIR5686, Society of Automotive Engineers, 2007.

${ }^{7}$ Chiereghin, N., MacManus, D., Savill, M. Dupuis, R, "Dynamic Distortion Simulations for Curved Aeronautical Intakes”, Advanced Aero Concepts, Design and Operation, Bristol, UK, 2014.

${ }^{8}$ S-16 Turbine Engine Inlet Flow Distortion Committee, "Inlet Total Pressure Distortion Considerations for Gas Turbine Engines”, Report No. AIR1419, Society of Automotive Engineers, 1999.

${ }^{9}$ Dupuis, R., "Influence of Inlet Flow Conditions and Geometric Parameters on Dynamic Distortions for S-Shaped Aeronautic Intakes", MSc Thesis, Cranfield University, UK, 2014.

${ }^{10}$ Bissinger, N. N. and Breuer, T., "Basic Principles - Gas Turbine Compatibility - Intake Aerodynamics Aspects", Encyclopaedia of Aerospace Engineering, John Wiley \& Sons, Ltd, 2010.

${ }^{11}$ Doorne, C. W. H., and Westerweel, J., "Measurement of Laminar, Transitional and Turbulent Pipe Flow Using Stereoscopic-PIV" Experiments in Fluids, Vol. 42, Dec. 2006, pp. 259-279.

${ }^{12}$ Nelson, M., Lowe, K. T., O'Brien, W. F., and Hoopes, K. M., "Stereoscopic PIV Measurements of Swirl Distortion on a Full-Scale Turbofan Engine Inlet”, 52 ${ }^{\text {nd }}$ AIAA Aerospace Sciences Meeting, National Harbor, Maryland, USA, 2014.

${ }^{13}$ Roach, P., E., "The Generation of Nearly Isotropic Turbulence by Means of Grids", International Journal of Heat and Fluid Flow, Vol. 8, no. 2, 1987, pp. 82-92.

${ }^{14}$ Raffel, M., Willert, C.E., Wereley, S.T., Kompenhans, J., Particle image Velocimetry $-A$ Practical Guide, $2^{\text {nd }}$ ed., Springer-Verlag Berlin, 2007.

${ }^{15}$ Seddon, J., Intake aerodynamics, 2nd edition, Blackwell Science, Oxford, 1999.

${ }^{16}$ Doane, D., P., Seward, L., E.,“Measuring Skewness: A Forgotten Statistic?”, Journal of Statistics Education, vol. 19, no. 2, 2011. 
2015-06-19

Flow distortion measurements in convoluted aero engine intakes

Zachos, Pavlos K.

AIAA

Zachos PK, MacManus DG and Chiereghin N. Flow distortion measurements in convoluted aero engine intakes. In: 33rd AIAA Applied Aerodynamics Conference, Dallas, TX., 22-26 June 2015 https://doi.org/10.2514/6.2015-3305

Downloaded from Cranfield Library Services E-Repository 